\begin{tabular}{|c|c|}
\hline Title & $\begin{array}{l}\text { Mechanism of formation, structural characteristics and photocatalytic activities of hierarchical-structured bismuth- } \\
\text { tungstate particles }\end{array}$ \\
\hline Author(s) & Hori, H.; Takase, M.; T akashima, M.; A mano, F.; Shibay ama, T.; Ohtani, B. \\
\hline Citation & $\begin{array}{l}\text { Catalysis today, 300, 99-111 } \\
\text { https://doi.org/10.1016/.cattod.2017.03.002 }\end{array}$ \\
\hline Issue Date & 2018-02-01 \\
\hline Doc URL & http:/hdl.handle.net/2115/76672 \\
\hline Rights & $\begin{array}{l}\text { () 2018. This manuscript version is made available under the CC-BY-NC-ND } 4.0 \text { license } \\
\text { http://reativecommons.org/icenses/by-nc-nd/4.0/ }\end{array}$ \\
\hline Rights(URL) & http://reativecommons.org/icenses/by-nc-nd/4.0/ \\
\hline Type & article (author version) \\
\hline File Information & BTSTF.pdf \\
\hline
\end{tabular}

Instructions for use 
[Special Issue on Photoactive Mixed Metal Oxides]

\title{
Mechanism of formation, structural characteristics and photocatalytic activities of hierarchical-structured bismuth-tungstate particles
}

\author{
H. Hori, ${ }^{1}$ M. Takase, ${ }^{2}$ M. Takashima,${ }^{1,3}$ F. Amano, ${ }^{4}$ T. Shibayama ${ }^{5}$ and B. Ohtani ${ }^{1,3 *}$ \\ ${ }^{1}$ Graduate School of Environmental Science, Hokkaido University, Sapporo 060-0810, Japan \\ ${ }^{2}$ Graduate School of Engineering, Muroran Institute of Technology, Muroran 050-8585, Japan \\ ${ }^{3}$ Institute for Catalysis, Hokkaido University, Sapporo 001-0021, Japan \\ ${ }^{4}$ Graduate School of Environmental Engineering, The University of Kitakyushu, Kitakyushu, 808- \\ 0135, Japan
}

${ }^{5}$ Center for Advanced Research of Energy Conversion Materials, Hokkaido University, Sapporo 0608628, Japan

*Corresponding author. E-mail address: ohtani@cat.hokudai.ac.jp (B. Ohtani)

Keywords:

Hierarchical flake-ball structure; Hollow particle; Bismuth oxyhydroxide; Core shell-structured droplet; Reaction at the boundary

\section{ABSTRACT}

The mechanism of the formation of flake ball-shaped (FB) bismuth-tungstate (BWO) particles was studied with samples prepared by hydrothermal reactions under standard conditions (tungsten/bismuth ratio of 0.55 in feed; $433 \mathrm{~K} ; 20 \mathrm{~h}$ ) and other modified conditions, and the structural characteristics of the samples were analyzed by powder X-ray diffraction (composition and crystal structure), field emission-type scanning electron microscopy (FE-SEM) (morphology), focused ionbeam etching followed by FE-SEM (inner void structure), ultrahigh-voltage transmission electron microscopy (inner void structure) and low-voltage scanning electron microscopy with energydispersive X-ray spectroscopic analysis (morphology and elemental distribution). Based on the results, a novel two-step mechanism of (1) formation of aqueous bismuth-oxyhydroxide droplets covered by tungstic acid (BOx@HWO) with fluidity and (2) reaction of inner bismuth and outer tungsten components at the BOx@HWO surface to yield BWO flakes is proposed as a one of the most plausible pathways that are consistent with the hollow structure of FB BWO and observations of the products prepared with shorter hydrothermal reaction. Photocatalytic activities of FB BWO samples in relation to their structure were studied.

\section{Introduction}

It does not seem necessary to point out the importance of metal oxides as photocatalysts, one 
of the most extensively studied applications of photoactive materials. Studies on metal-oxide photocatalysis started with the use of simple metal oxides (SMOs) such as titanium(IV) oxide $\left(\mathrm{TiO}_{2}\right.$ ) and zinc oxide $(\mathrm{ZnO})$ [1-4], but a limitation of the use of SMOs as visible light-responsive photocatalysts had already been shown in the 1980's; Scaife pointed out that the conduction-band (CB) bottom position of an SMO is lowered (becoming more anodic) by reducing the band-gap energy, i.e., photocatalytic hydrogen evolution cannot be achieved using visible light-absorbing SMOs because the valence band (VB) of SMOs is commonly composed of oxygen $2 p$ orbitals and therefore the VB top positions are almost the same regardless of the kind of metals in metal oxides [5]. Two main strategies have been used to solve this problem, i.e., to make a material absorb visible light while keeping the CB-bottom position sufficiently cathodic. One strategy is doping heteroatoms in wide band-gap SMOs such as $\mathrm{TiO}_{2}$ to raise the VB-top position by expanding the VB upward [6,7], and the other strategy is using mixed metal oxides (MMOs; including metal metallates, which are not included in MMOs in a strict scientific sense) having a VB composed of oxygen and metal orbitals [8,9]; the Scaife's paper had already shown that several visible light-absorbing MMOs had a CB-top position that was more cathodic than the proton/hydrogen standard electrode potential [5].

In parallel to (or at the same time as) those successful studies on SMO/MMOs with a desirable band structure, i.e., studies to find SMO/MMOs with appropriate composition/crystal structures, studies were being conducted on the effect of photocatalyst-particle morphology on photocatalytic activities. In one of the pioneering works, ion-exchanged potassium niobate $\left(\mathrm{K}_{4} \mathrm{Nb}_{6} \mathrm{O}_{17}\right)$ with a layered crystal structure was used to separate the reaction sites for reduction and oxidation by photoexcited electrons and positive holes, respectively [10]. A recent trend in studies on morphology-dependent photocatalytic activity has been the use of faceted-crystalline SMO/MMO particles such as anatase $\mathrm{TiO}_{2}$ [11-14] and bismuth vanadate $\left(\mathrm{BiVO}_{4}\right)$ [15,16]. Among MMOs, many metal metallates (oxymetallates) such as $\mathrm{K}_{4} \mathrm{Nb}_{6} \mathrm{O}_{17}$ and bismuth tungstate $\left(\mathrm{Bi}_{2} \mathrm{WO}_{6}\right.$; $\mathrm{BWO}$ ) have a layered crystal structure, which makes it possible to fabricate a variety of morphologically controlled particles by ion exchange [17,18], exfoliation and reconstruction [19] or assembling crystalline platelets in different ways as described below.

As was reported in papers from our laboratory [20-23], hierarchically structured flake ballshaped (FB) BWO particles with outer diameters of ca. 3-5 $\mu \mathrm{m}$ have been prepared under controlled hydrothermal (HT) conditions. The hierarchical structure was composed of (i) BWO crystalline nanoplates, (ii) flakes of stacked layered assemblies of the nanoplates, and (iii) spherical assemblies of flakes as primary, secondary and ternary phases, respectively (Fig. 1).

(Fig. 1)

Although there is still discussion on the effect of this unique hierarchical structure on photocatalytic activity, FB BWO seemed to show higher activities than those of BWO without the characteristic structure. Since the crystal phase of BWO, russellite [24], has a layered structure, particles prepared in an ordinary way, e.g., solid-state reaction of bismuth oxide and tungsten oxide, are stacked plates or particles exposing flat surfaces (Fig. 1 (d) [20] and Fig. S2 [25]) reflecting the layered crystal structure 
of russellite. The FB structure must therefore be regulated by during its HT synthesis. As was shown in previous papers [21,23], possible key parameters for production of FB-shaped particles, notstacked platelet crystallites, are an acidic condition ( $\mathrm{pH}$ ca. 1) and a $10 \mathrm{~mol} \%$-excess amount of the tungsten source (sodium tungstate; $\mathrm{Na}_{2} \mathrm{WO}_{4}$ ). The former condition might inhibit the stacking of platelet crystallites and the latter condition might lead to the formation of spherical particles (BF) with a homogeneous size distribution; as has been reported by the authors' group [21] and Zhang et al. $[26,27]$. HT reaction of a stoichiometric mixture of the bismuth source $\left(\mathrm{Bi}\left(\mathrm{NO}_{3}\right)_{3}\right)$ and tungsten source $\left(\mathrm{Na}_{2} \mathrm{WO}_{4}\right)$ in an acidic suspension gave FB particles and partially spherical pseudo-FB particles (defined as spherical and broken "flower-like" structures, respectively, in the papers by Zhang et al. [26]). In the present study, preparation conditions were fixed to those reported FB-producing ones, and FB BWO particles were obtained without exception. Then, it must be significant to understand the actual mechanism of FB BWO-particle production under the controlled conditions [25]. The purpose of this paper is to clarify the detailed mechanism of unique FB BWO-particle formation and the important parameters controlling the quality of FB BWO particles and to propose a novel strategy for control of particle morphology.

\section{Experimental}

\subsection{Procedure for sample preparation}

FB BWO particles were prepared by HT reaction according to previous works [20-23] using a reaction mixture of bismuth nitrate $\left(\mathrm{Bi}\left(\mathrm{NO}_{3}\right)_{3}\right)$ pentahydrate (Wako Pure Chemical) and sodium tungstate $\left(\mathrm{Na}_{2} \mathrm{WO}_{4}\right)$ dihydrate (Wako Pure Chemical) in water. A standard procedure for preparation of FB BWO particle with a tungsten-to-bismuth molar ratio in feed (W/Bi) of 0.55 is as follows. The bismuth source $\left(\mathrm{Bi}\left(\mathrm{NO}_{3}\right)_{3} \cdot 5 \mathrm{H}_{2} \mathrm{O} ; 2.41 \mathrm{~g} ; 5.0 \mathrm{mmol}\right.$ ) was added to a $10 \mathrm{~mL}$ of Milli-Q water (Water System, Ultrapure, Millipore Synergy with UV; $18.2 \mathrm{M} \Omega \mathrm{cm}$ ) in a flask. The resultant white suspension was stirred vigorously by a magnetic stirrer for $10 \mathrm{~min}$, and then a 40-ml aqueous (MilliQ) solution of the tungsten source $\left(\mathrm{Na}_{2} \mathrm{WO}_{4} \cdot 2 \mathrm{H}_{2} \mathrm{O} ; 0.907 \mathrm{~g} ; 2.75 \mathrm{mmol} ; \mathrm{W} / \mathrm{Bi}=0.55\right)$ was added dropwise over a period of ca. 20 min with vigorous magnetic stirring. When a different $\mathrm{W} / \mathrm{Bi}$ ratio was used, the amount of $\mathrm{Na}_{2} \mathrm{WO}_{4} \cdot 2 \mathrm{H}_{2} \mathrm{O}$ was adjusted. After further magnetic stirring for $10 \mathrm{~min}$ and sonication for $10 \mathrm{~min}$ using a Yamato-Branson 5510 ultrasonic cleaner (42 kHz; $180 \mathrm{~W})$, the suspension and washing were placed in a Teflon (PTFE) bottle with a Teflon lid (San-ai Science HUT100 ), and the total volume of the suspension was adjusted to ca. $70 \mathrm{~mL}$ to leave a head space of ca. 27 $\mathrm{mL}$. If necessary, $\mathrm{pH}$ of a feed suspension was measured using a D-72T Horiba portable $\mathrm{pH}$ meter with a $9680 \mathrm{~S}$ glass electrode calibrated with buffer solutions $(\mathrm{pH}=1.68$ and 6.86). Into a $433 \mathrm{~K}$ preheated oven was placed a stainless outer bottle (San-ai Science HU-100) incorporating the reaction mixture in the sealed Teflon container and it was heated without agitation or stirring for $20 \mathrm{~h}$. The reaction bottle was allowed to cool in the switched-off oven to room temperature for ca. $10 \mathrm{~h}$ in the standard procedure or the bottle was taken out of the oven after a certain period (2.25-15.0 h) of heating and quenched in ice-cold water. The reaction mixture was taken from the container, and a slightly 
yellow solid product was recovered by centrifugation at $3000 \mathrm{rpm}$, washed with Milli-Q water at least three times, and dried at $393 \mathrm{~K}$ in air for at least $4 \mathrm{~h}$.

\subsection{Composition analysis using X-ray diffractometry and thermogravimetry}

Crystalline (and non-crystalline) composition of samples was analyzed by X-ray diffractometry (XRD) and thermogravimetry. In XRD measurement using a Rigaku SmartLab diffractometer, $0.300 \mathrm{~g}$ of a sample and $0.075 \mathrm{~g}$ of nickel oxide (NiO; Wako Pure Chemical) as an internal standard were mixed thoroughly in an agate mortar and set in a sample holder. The measurement conditions were as follows: an X-ray tube (copper $\mathrm{K}_{\alpha}$ ) operated at $40 \mathrm{kV}$ and $30 \mathrm{~mA}$; scanning rate of $1.0^{\circ} \mathrm{min}^{-1}$; step of $0.02^{\circ}$; and $2 \theta$ range of $3-80^{\circ}$. The acquired diffractograms were analyzed by software installed in the controlling personal computer (PDXL including a RIETAN-FP Rietveld analysis package). Based on the assumption that $\mathrm{NiO}$ is $100 \%$ crystalline, i.e., without a non-crystalline part, crystalline BWO content was calculated from the results of Rietveld analysis [28]. Water content in the samples was analyzed by thermogravimetry using an Advance Riko TGD-9700 thermogravimetry/differential thermal analysis instrument with an alumina sample holder under constant dry-air flow in the temperature range of 293-793 K. The content of water in samples was estimated with the assumption that the weight loss by heating corresponds solely to water release.

\subsection{Sonication and alkaline treatment}

As post-treatment of samples, sonication was performed for a suspension of the product after HT reaction in ca. $50 \mathrm{~mL}$ of an original acidic supernatant, Milli-Q water or $1 \mathrm{~mol} \mathrm{~L}^{-1}$ aqueous solution of sodium hydroxide $(\mathrm{NaOH})$. In the latter two cases, the HT product was washed three times before addition of water or aqueous $\mathrm{NaOH}$. The suspension in a $50-\mathrm{mL}$ glass tube was sonicated using a Yamato-Branson 5510 ultrasonic cleaner $(42 \mathrm{kHz} ; 180 \mathrm{~W})$ for $90 \mathrm{~min}$. The solid sample was washed with Milli-Q water a few times, except for the neutral suspension, and then recovered by centrifugation and dried in air at $393 \mathrm{~K}$.

Alkaline post-treatment was performed for a suspension of the product after the abovementioned HT reaction with ca. $50 \mathrm{~mL}$ of aqueous $\mathrm{NaOH}\left(1 \mathrm{~mol} \mathrm{~L}^{-1}\right)$ in a $50-\mathrm{mL}$ sample tube without stirring for $8.5 \mathrm{~h}$. After treatment, the solid sample was washed with $50 \mathrm{~mL}$ of Milli-Q water three times and dried at $393 \mathrm{~K}$ in air.

\subsection{Scanning electron microscopy}

Size and morphology of the products were analyzed by field-emission scanning electron microscopy (FE-SEM, JEOL JSM-7400F) in low secondary-electron image (LEI) mode with 2.0-5.0 $\mathrm{kV}$ electron-acceleration voltage, $10.0-\mu \mathrm{A}$ current and 6-mm working distance. A small amount of the sample powder was fixed on a brass sample stage (12 $\mathrm{mm}$ in diameter; $10 \mathrm{~mm}$ in height) using conductive carbon tape (Okenshoji \#15-1096) and dried under reduced pressure ( $<100 \mathrm{~Pa})$ overnight. For counting the number fraction of FB BWO particles and for measuring the flake thickness, at least 500 particles and 100 flakes were checked in several SEM images, respectively. 
For low-voltage scanning electron microscopy (LV-SEM) analysis, samples prepared by quenching followed by washing with Milli-Q water were used without drying. The aqueous suspension was poured onto a sheet of aluminum foil and dried at room temperature in air to leave a solid tightly adhered to the aluminum sheet. Two methods were used to fix the sample on a brass sample stage (same as that used in the above-mentioned FE-SEM analysis). One method was pressing a stage with conductive carbon tape on the solid-adhered aluminum sheet (back-side sampling). In the other method, the solid was peeled off with a piece of adhesive tape and then transferred to conductive carbon tape on a stage (front-side sampling). LV-SEM (JEOL JSM6360LA) with energy-dispersive X-ray spectroscopic (EDS) analysis was performed under the conditions of 10-mm working distance, $15-\mathrm{kV}$ electron-acceleration voltage, 1.0-nA current and 0-20 $\mathrm{keV}$ energy range for EDS.

\subsection{Focused ion-beam etching and cross-sectional surface observation}

Specimens for cross-sectional surface observation were prepared by etching FB BWO particles prepared by HT reaction with standard conditions (W/Bi $=0.55 ; 433 \mathrm{~K} ; 20 \mathrm{~h})$ via focused ion beam (FIB) milling using an accelerated gallium ion at $30 \mathrm{kV}$ (performed on a Micrion JFIB-2100 instrument). The thus-exposed cross-sectional surfaces were observed by a field-emission scanning electron microscope (JEOL JSM-7000FA) in secondary-electron imaging (SEI) mode with 5.0-kV electron-acceleration voltage and 15-mm working distance.

\subsection{Ultra-high voltage transmission electron microscopic analysis}

Solid samples prepared by HT reaction with standard conditions $(\mathrm{W} / \mathrm{Bi}=0.55 ; 433 \mathrm{~K} ; 20 \mathrm{~h})$ or with short HT durations and subsequent quenching were fixed on Okenshoji NS-C15 microgrids and dried under reduced pressure $(<100 \mathrm{~Pa})$ overnight. The samples were observed by a multi-beam high voltage electron microscope (JEOL JEM-ARM-1300) at electron-accelerating voltage of 1250 $\mathrm{kV}$.

The thickness of electron-beam transmission $(L)$ in arbitrary units was estimated by densitometry of black-and-white TEM images on the basis of the Lambert-Beer law for electron-beam absorption:

$$
L=\log \left(I_{0} / I\right)=\log \left\{D_{\max } /\left(D_{\max }-D\right)\right\},
$$

where $I_{0}$ and $I$ are intensities of the electron beam before and after passage of a sample, respectively, and $D_{\max }$ and $D$ are maximum density and density of a given position in a TEM image, respectively. Simulation of the thickness profile was performed by assuming a hollow particle with a shell of homogeneous density.

\subsection{Photocatalytic activity test}

As one of the representative photocatalytic reactions, carbon-dioxide $\left(\mathrm{CO}_{2}\right)$ liberation from aqueous acetic acid under aerobic conditions was used for a photocatalytic activity test [29-31]. 
Among four representative photocatalytic-activity test reactions [31] (methanol dehydrogenation, oxygen evolution along with silver-metal deposition, gas phase oxidative decomposition of acetaldehyde and the present acetic-acid decomposition) frequently used in the authors' laboratory, methanol dehydrogenation requires in-situ deposition of platinum, which is also influenced by the properties of the photocatalyst [32,33], oxygen-evolution rate is predominantly controlled by silverion adsorption and particle size [31] and acetaldehyde decomposition is mainly regulated by specific surface area [22]. Thus, acetic acid decomposition seems most appropriate for first-step photocatalytic activity test. A brief interpretation of the experimental procedures is as follows. A $50-\mathrm{mg}$ portion of the powder sample was suspended in a 5.0vol\% aqueous acetic acid solution (5.0 $\mathrm{mL}$ ) in a borosilicate glass tube (transparent for light of wavelength $>290 \mathrm{~nm}, 18 \mathrm{~mm}$ in inner diameter and $180 \mathrm{~mm}$ in length), and the tube was tightly sealed using a double-capped rubber septum and a sheet of Parafilm. The sample was irradiated in a thermostated bath $(298 \mathrm{~K})$ by a $400-\mathrm{W}$ highpressure mercury arc (Eiko-sha) under 1000-rpm magnetic stirring. The product $\mathrm{CO}_{2}$ was monitored using a Shimadzu GC-8A gas chromatograph equipped with a TCD and a Porapak-Q column. Photocatalytic activities were calculated and are presented as rates of $\mathrm{CO}_{2}$ liberation.

\section{Results and Discussion}

\subsection{Unique shape of flake-ball particles with a hierarchical structure}

In our previous report [21], it was suggested that BWO flakes are first produced independently in the early stage of the HT process and then they are assembled to be FB particles, though small BWO flakes had not been observed by short-term $(2 \mathrm{~h})$ hydrothermal reaction; small FB BWO particles were obtained instead. Another inconsistency was the structure of flakes, i.e., stacked nanosheets of BWO; it seems that there are spaces between BWO nanosheets because the presence of micropores (nanometer-sized spaces) in FB BWO was suggested by analysis of nitrogen adsorption behavior [34] and wet milling of FB BWO particles led to disappearance of those micropores. In other words, the second-phase structure, flakes, does not seem to be assemblies of the first-phase structure, plates. Taking into consideration the fact that stacked platelet crystallites, not FB BWO, are also formed under the above-mentioned higher-pH conditions, the formation of FB BWO might be due to the inhibition of the plate stacking or assembling of flakes with homogeneous-sized spherical particles as a core.

\subsection{Initial composition and structure before HT reaction in FB-BWO preparation}

FB BWO particles were prepared by hydrothermal reaction of a mixture of bismuth nitrate $\left(\mathrm{Bi}\left(\mathrm{NO}_{3}\right)_{3} \cdot 5 \mathrm{H}_{2} \mathrm{O}\right)$, sodium tungstate $\left(\mathrm{Na}_{2} \mathrm{WO}_{4}\right)$ and water. First, the bismuth source, $\mathrm{Bi}\left(\mathrm{NO}_{3}\right)_{3} \cdot 5 \mathrm{H}_{2} \mathrm{O}$ $(5.0 \mathrm{mmol})$, was added to Milli-Q water $(10 \mathrm{~mL})$ at ambient temperature to yield a white suspension due to hydrolysis; unstable actual $\mathrm{pH}$ of the suspension suggests an incomplete or slow equilibrium.

(Table 1)

Assuming complete hydrolysis of bismuth nitrate, 


$$
\mathrm{Bi}\left(\mathrm{NO}_{3}\right)_{3}+3 \mathrm{H}_{2} \mathrm{O} \rightarrow \mathrm{Bi}(\mathrm{OH})_{3}+3 \mathrm{HNO}_{3},
$$

with negligible dissolution of bismuth hydroxide $\left(\mathrm{Bi}(\mathrm{OH})_{3}\right)$, $\mathrm{pH}$ should be zero, which is lower than the actual $\mathrm{pH}$ (0.27; entry 1 in Table 1), indicating incomplete hydrolysis; almost complete proton dissociation of nitric acid at the same concentration was confirmed by a separate experiment (entry 2 in Table 1). Previous studies on bismuth-nitrate hydrolysis suggested the formation of $\left[\mathrm{Bi}_{6} \mathrm{O}_{5}(\mathrm{OH})_{3}\right]\left(\mathrm{NO}_{3}\right)_{5}(\mathrm{BO})$ [35] or $\left[\mathrm{Bi}_{6} \mathrm{O}_{4}(\mathrm{OH})_{4}\right]\left(\mathrm{NO}_{3}\right)_{6}(\mathrm{BO} 4)$ [36] with smaller nitric-acid release.

$$
\begin{aligned}
& 6 \mathrm{Bi}\left(\mathrm{NO}_{3}\right)_{3}+8 \mathrm{H}_{2} \mathrm{O} \rightarrow\left[\mathrm{Bi}_{6} \mathrm{O}_{5}(\mathrm{OH})_{3}\right]\left(\mathrm{NO}_{3}\right)_{5}+13 \mathrm{HNO}_{3} \\
& 6 \mathrm{Bi}\left(\mathrm{NO}_{3}\right)_{3}+8 \mathrm{H}_{2} \mathrm{O} \rightarrow\left[\mathrm{Bi}_{6} \mathrm{O}_{4}(\mathrm{OH})_{4}\right]\left(\mathrm{NO}_{3}\right)_{6}+12 \mathrm{HNO}_{3}
\end{aligned}
$$

Even if those partial hydrolysis processes are assumed, actual $\mathrm{pH}(0.27)$ seems still to be a little low. Therefore, it should be presumed that other partially hydrolyzed forms remained in the suspension. In other words, $\mathrm{Bi}(\mathrm{OH})_{3}$ was partially neutralized by nitric acid. Actually, solid samples containing BO5 and BO4 were obtained by drying up of the pH-regulated suspensions (entry 5 in Table 1).

The tungsten source, sodium tungstate $\left(\mathrm{Na}_{2} \mathrm{WO}_{4} ; 2.75 \mathrm{mmol}(10 \mathrm{~mol} \%\right.$ excess $)$ ) was dissolved in Milli-Q water $(40 \mathrm{~mL})$, and the solution was added dropwise to the above-mentioned bismuth species-containing suspension to give a white suspension $(70 \mathrm{~mL})$ of $\mathrm{pH}=1.1$ (entry 5 in Table 1). Slightly basic $\mathrm{pH}$ of the $\mathrm{Na}_{2} \mathrm{WO}_{4}$ solution (7.8-7.9; entry 3 in Table 1) suggests that tungstic acid $\left(\mathrm{H}_{2} \mathrm{WO}_{4}\right.$; $\left.\mathrm{HWO}\right)$ is almost completely proton-dissociated to be tungstate $\left(\mathrm{WO}_{4}{ }^{2-}\right)$, and this is consistent with the fact that the sodium-tungstate solution is stable after standing for a long time without giving a precipitate of tungsten oxide; HWO has relatively high acidity, i.e., low $\mathrm{p} K_{\mathrm{a}}$ as an acid [37]. Upon addition of the $\mathrm{Na}_{2} \mathrm{WO}_{4}$ solution to the bismuth species-containing suspension, $\mathrm{pH}$ of the suspension was raised to 1.1 with 2.75 or $2.5 \mathrm{mmol}$ sodium tungstate. The increase of $\mathrm{pH}$ is explained by release of hydroxide anions by hydrolysis of tungstate anions:

$$
\mathrm{WO}_{4}{ }^{2-}+2 \mathrm{H}_{2} \mathrm{O} \rightarrow \mathrm{H}_{2} \mathrm{WO}_{4}+2 \mathrm{OH}^{-} .
$$

It has been reported that partial hydrolysis of tungstate occurs at $\mathrm{pH} \mathrm{1-2} \mathrm{to} \mathrm{give} \mathrm{soluble} \mathrm{tungstate}$ anions [37], as

$$
\begin{gathered}
12 \mathrm{WO}_{4}^{2-}+10 \mathrm{H}_{2} \mathrm{O} \rightarrow\left[\mathrm{H}_{2} \mathrm{~W}_{12} \mathrm{O}_{40}\right]^{6-}+18 \mathrm{OH}^{-} \text {and } \\
11 \mathrm{WO}_{4}{ }^{2-}+12 \mathrm{H}_{2} \mathrm{O} \rightarrow\left[\mathrm{H}_{8} \mathrm{~W}_{11} \mathrm{O}_{40}\right]^{6-}+16 \mathrm{OH}^{-},
\end{gathered}
$$

though such partial hydrolysis giving less than two hydroxide anions from one tungstate anion cannot explain the suspension $\mathrm{pH}$ that is slightly higher than that expected even assuming complete hydrolysis of tungstate anions. Thus, the tungsten source might be in the form of HWO or hydrated tungsten oxide $\left(\mathrm{WO}_{3} \cdot \mathrm{nH}_{2} \mathrm{O}\right)$. In a control experiment (entry 4 in Table 1), a yellow precipitate (presumably so-called "yellow tungstic acid"; $\mathrm{WO}_{3} \cdot \mathrm{H}_{2} \mathrm{O}$ ) was observed in a sodium tungstate solution when the solution $\mathrm{pH}$ was controlled to be 1.1, the same as that of the reaction suspension (entry 5 in Table 1), with an appreciable (ca. $10 \mathrm{~min}$ ) induction period. On the other hand, the actual reaction suspension remained white before and after HT reaction, indicating negligible formation of yellow tungstic acid. 
There are two possible reasons. One possible reason is that $\mathrm{HWO}\left(\right.$ or $\mathrm{WO}_{3} \cdot \mathrm{H}_{2} \mathrm{O}$ ) is deposited on the surface of bismuth oxyhydroxides in a highly dispersed or thin-layer form. The other possibility is reaction of $\mathrm{HWO}$ with $\mathrm{Bi}(\mathrm{OH})_{3}$ (or bismuth oxyhydroxide, $\mathrm{BO} 4$ and $\mathrm{BO}$ ) to liberate bismuth oxyhydroxide like $\mathrm{BO} 4$ or $\mathrm{BO}$. In the latter case, assuming that a $\mathrm{BO} 4-$ like reaction proceeds, the molar $\mathrm{Bi} / \mathrm{W}$ ratio in the oxyhydroxide is 2 and, therefore, $\mathrm{Bi}(\mathrm{OH})_{3}$ should be thoroughly converted if all of the tungstic acid is reacted. However, acid strength of tungstic acid must be weaker than that of nitric acid, and it therefore seems less probable that the tungsten source is present as a counter anion of bismuth oxyhydroxide cation. On the basis of these considerations, HWO might be deposited on the bismuth oxyhydroxides, BO5 and BO4 (BOx), in the suspension before $\mathrm{TH}$.

Based on the assumption that the tungsten source was present in the form of HWO (or $\left.\mathrm{WO}_{3} \cdot \mathrm{nH}_{2} \mathrm{O}\right)$, the amount of nitric acid released in the suspension containing bismuth nitrate $(5.0 \mathrm{mmol})$ and sodium tungstate $(2.75 \mathrm{mmol})$ is estimated to be 5.6 (entry 5 in Table 1), which corresponds to ca. $60 \%$ molar amount of nitric acid released by possible complete hydrolysis of $\mathrm{Bi}\left(\mathrm{NO}_{3}\right)_{3}$ (eq. 2). Since partial hydrolysis with eqs. 3 and 4 releases $72 \%$ and $67 \%$ molar amount of nitric acid compared with complete hydrolysis, the white precipitate in the suspension before HT reaction might be partially hydrolyzed bismuth oxyhydroxides, $\mathrm{BOx}$, which might be covered by $\mathrm{HWO}\left(\right.$ or $\mathrm{WO}_{3} \cdot \mathrm{nH}_{2} \mathrm{O}$ ) as mentioned above. In fact, XRD analysis of powder recovered from the reaction mixture without the HT process (entry 5 in Table 1) revealed the presence of BO4 (59\% in total crystalline components), BO5 (10\%) and HWO. The Bi/W ratio in those crystallites was calculated to be 1.87 , which is reasonably close to the theoretical value $(1.82=5.0 / 2.75)$ considering that there might be amorphous components giving no XRD peaks.

\subsection{Composition and crystalline structure of the final product FB BWO}

The stoichiometry of the reaction of $\mathrm{FB}$ BWO production from $\mathrm{Bi}\left(\mathrm{NO}_{3}\right)_{3}$ and $\mathrm{Na}_{2} \mathrm{WO}_{4}$ is formally shown as follows.

$$
2 \mathrm{Bi}\left(\mathrm{NO}_{3}\right)_{3}+\mathrm{Na}_{2} \mathrm{WO}_{4}+2 \mathrm{H}_{2} \mathrm{O} \rightarrow \mathrm{Bi}_{2} \mathrm{WO}_{6}+4 \mathrm{HNO}_{3}+2 \mathrm{NaNO}_{3}
$$

Since it is expected that $10 \mathrm{~mol} \%$-excess $\mathrm{Na}_{2} \mathrm{WO}_{4}$ works as a base, $9.5(=5.0 \times 3-2.75 \times 2) \mathrm{mmol}$ nitric acid may be released and remain in the suspension after HT reaction. As shown in Table 1 (entry 6), the amount of acid in the final suspension was estimated to be $7.9 \mathrm{mmol}$, corresponding to $83 \%$ of the expected value; a control experiment (entry 10 in Table 1) of partial neutralization revealed that nitric acid concentration can be estimated using $\mathrm{pH}$. This indicates that the reaction (eq. 8) was not completed, since a control experiment (entry 11 in Table 1) suggested negligible adsorption of nitric acid on the surface of FB BWO; $\mathrm{pH}$ of the suspension reproducing the ideal reaction mixture after complete conversion to BWO (eq. 8; entry 11 in Table 1) was almost the same as that of a solution containing nitric acid and sodium hydroxide (entry 10 in Table 1). The fact that the product containing FB BWO included still $20 \%$ of non-crystalline components (amorphous solid and water) is consistent with the above-mentioned speculation. Thus, a small part of the bismuth source, partially hydrolyzed bismuth nitrate, i.e., bismuth oxyhydroxides (BOx), as well as HWO, might remain in the 
product even after a long HT process.

\subsection{Macroscopic structure of FB BWO particles}

In the preceding paper, we reported that there are hollow spaces covered by the flakes in FB BWO particles prepared with $\mathrm{W} / \mathrm{Bi}=0.55$ [25]. There has been evidence supporting this hollow structure. The evidence includes the observation of partially broken particles with inner void spaces; sonication of FB BWO particles led to the breakage of $\mathrm{FB}$ particles $(\mathrm{W} / \mathrm{Bi}=0.55)$ to show inner void spaces regardless of the sonication conditions, acidic, neutral or basic conditions [25]. As shown in SEM images (Fig. 2), sonication of once-prepared FB BWO particles (W/Bi $=0.50)$ under acidic, neutral and basic conditions for $90 \mathrm{~min}$ also led to breakage of FB particles to show inner void spaces regardless of $\mathrm{W} / \mathrm{Bi}$.

(Fig. 2)

Although a certain compound might exist in FB particles as a core and be dissolved by sonication to leave void spaces, the fact that void spaces in FB particles were observed regardless of $\mathrm{pH}$ of the sonication suspension and initial W/Bi suggests that those void spaces were there in the original FB BWO particles. Another finding in the post-treatment of once-prepared FB BWO particles was thinning of flakes by alkali treatment. As Fig. 3 shows, the average thickness of flakes of alkali (sodium hydroxide)-treated particles $(\mathrm{W} / \mathrm{Bi}=0.55)$ seemed to be reduced $(\mathrm{ca} .40 \%)$.

(Fig. 3)

Since such flake thinning was not evident in FB BWO particles prepared with $\mathrm{W} / \mathrm{Bi}=0.50$, i.e., the thickness was slightly smaller than that of $\mathrm{W} / \mathrm{Bi}=0.55$ particles, an excess tungsten component might be present between the plates, though further study is needed to clarify the location of the excess tungsten component.

To confirm the hollow structure of FB BWO, independent measurements using destructive focused ion beam (FIB) milling and non-destructive ultrahigh-voltage transmission electron microscopy (UHV-TEM) were performed. Figure 4 shows FIB-milled FB BWO particles.

(Fig. 4)

The front parts of two FB BWO particles were milled off by downdraft ion beams to expose cross sections of the particles. Shrinkage due to the heat by FIB milling might occur to give a smaller particle size and densification, especially in the outer part, but it is clear that there were void spaces (or low-density parts) in the core part. The size of void space seemed to be $1-1.5 \mu \mathrm{m}$, almost the same as or slightly smaller than that estimated from SEM images in shown Fig. 2, at least partly because of heat shrinkage during FIB. Figure 5 shows the results of non-destructive UHV-TEM analysis.

(Fig. 5)

Even at the electron-acceleration voltage of $1250 \mathrm{kV}$, an electron beam could not pass through FB 
BWO particles larger than $2 \mu \mathrm{m}$, but transmission images could be obtained for smaller particles. Assuming homogeneous electron-beam extinction at the solid part of samples, the thickness was estimated by densitometry of black-and-white TEM images to show void spaces inside, i.e., hollow structure in FB BWO particles prepared under the standard HT conditions. Simulated thickness profiles based on the assumption of an ideal hollow structure composed of a shell of constant density (Fig. 5) could not be fitted to the observed profiles. There are a few reasons for this discrepancy. One is the inhomogeneous density of an FB part of particles as suggested by the zig-zag patterns of thickness at the center, and transmission might occur between BWO flakes. Another reason is the possible large experimental error; an electron could be absorbed almost completely at the highest density part of patterns and thereby estimated thickness might be saturated. Consequently, at the present stage, the authors cannot conclude the precise thickness profile of FB BWO particles, but the hollow structure could be confirmed. This is supported by TEM images of quenched samples with particle diameters smaller than $2 \mu \mathrm{m}$ (Fig. 6).

(Fig. 6)

It can be said that particles prepared by HT reaction of $140 \mathrm{~min}$ in duration and subsequent quenching, in which FB BWO had started to appear, did not contain a low-density part in their center even though the outer size was sufficiently small to observe those parts if present. On the other hand, low-density parts in the center of particles were frequently observed in particles prepared by 300-min and 600-min HT reactions and were almost always observed in particles prepared by $900-\mathrm{min}$ HT reaction. Although the UHV-TEM analyses were limited to samples with particle size smaller than $2 \mu \mathrm{m}$ and there is no information on 3-5 $\mu \mathrm{m}$-sized FB BWO particles, the results are supporting evidence for the absence of inner void spaces in intermediate particles at the beginning of the HT process.

On the basis of these considerations, it can be concluded that the hollow structure of FB BWO particles is predominantly composed of crystalline BWO starting from partially hydrolyzed bismuth nitrate (BOx) and HWO. The question thus arises as to how these hollow particles are produced during the HT reaction. In a previous paper, the authors suggested that bismuth-rich spherical solid particles are liberated first and then they are converted into hollow-structured particles by growth of BWO plate-like crystallites [25]. Thee time course of FB BWO-particle formation was thus studied.

\subsection{Time course of FB BWO-particle production}

Figure 7 shows SEM images of solid products recovered from the reaction mixture after HT reaction for 135, 140, 145 and 150 min followed by quenching in ice-cold water.

(Fig. 7)

Both FB particles and small particles were observed except for the 135-min sample, in which no FB particles were seen not only in this image but also in other SEM images of this 135-min sample. The proportion of small particles decreased with increase in HT duration and was almost zero after 20-h HT reaction. Thus, $\mu \mathrm{m}$-sized FB BWO particles seemed to appear suddenly at $140 \mathrm{~min}$. Results of 
XRD analyses of those products support this (Table 2); the solid product recovered after 140-min HT reaction contained 32\% BWO and the BWO-crystallite content was increased with increase in HT duration, while the solid product of 135-min HT reaction was almost amorphous. It is notable that a change in $\mathrm{pH}$ of the reaction mixture at HT duration between 135 and 140 min was not evident, which will be discussed later.

Figure 8 shows LV-SEM and EDS elemental-mapping images of the 135-min sample that contained no FB BWO particles as well as those images of an FB BWO particle.

\section{(Fig. 8)}

The sample was predominantly composed of nanoparticles that contained both bismuth and tungsten homogeneously within the EDS resolution (data not shown), but $\mu \mathrm{m}$-sized particles could be observed. Representative LV-SEM and elemental-mapping images of those particles in Fig. 8 show a "starfruitlike" morphology with sizes of 2-3 $\mu \mathrm{m}$ and a high concentration of bismuth as shown by the green color, respectively. The tungsten/bismuth atomic ratio in each image might depend on the method for specimen preparation; back-side sampling gave a ratio slightly higher than that by front-side sampling, suggesting that smaller nanoparticles containing both bismuth and tungsten tended to be preferentially accumulated on an aluminum sheet and larger starfruit-like particles are deposited on the nanoparticle layer when sampling. The starfruit-like morphology might be related to the trend of formation of angular-shaped crystallites when BOx was recovered by drying of a suspension containing BOx (entry 5 in Table 1) and a solid product obtained by an HT reaction in which sodium hydroxide was used instead of $\mathrm{Na}_{2} \mathrm{WO}_{4}$ (entry 9 in Table 1), as shown in Fig. 9.

(Fig. 9)

The former and latter were assigned (Table 1) to a mixture of BO4, HWO and BO5 and bismuth oxyhydroxide $\left(\mathrm{Bi}_{2} \mathrm{O}_{2}(\mathrm{OH})\left(\mathrm{NO}_{3}\right)\right.$; BO6) [38]. Similar SEM images have been reported for bismuth oxyhydroxide prepared by an $\mathrm{HT}$ reaction of an aqueous $\mathrm{Bi}\left(\mathrm{NO}_{3}\right)_{3}$ suspension at $\mathrm{pH} 3.5$ [39]. Those results suggest that the starfruit-like particles were of bismuth oxyhydroxide (BOx). A significant point is that no starfruit-like particles have been observed in SEM images of solid samples containing bismuth oxyhydroxides, such as BO6, BO5 and BO4, except for the 135-min HT product in the present study, suggesting that the starfruit-like particles cannot be produced from a precursor only containing a bismuth component.

\subsection{Possible mechanism of FB BWO-particle formation}

Based on the above-described results, the mechanism of FB BWO-particle formation is proposed as shown in Fig. 10 as a two-step reaction.

(Fig. 10)

The first step is the formation of spherical liquid droplets of a highly concentrated aqueous suspension/emulsion of BOx (or hydrated BOx having fluidity) covered by HWO (or its oligomer). Although no direct evidence for the existence of core shell-structured droplets (BOx@HWO), e.g., 
results of less possible in-situ size-distribution and elemental-composition analyses during HT reaction, has been obtained, almost all the results can be interpreted using a mechanistic model initiated with BOx@HWO formation. As discussed in Section 3.2., a reaction mixture before HT reaction contains BOx as a bismuth component. Since HT treatment of a suspension without $\mathrm{Na}_{2} \mathrm{WO}_{4}$ (entry 9 in Table 1; $\mathrm{pH}$ adjusted by adding sodium hydroxide instead of $\mathrm{Na}_{2} \mathrm{WO}_{4}$ ) produced aggregates of large irregularly-shaped but somewhat angular-shaped particles, the BOx@HWO formation must depend on the presence of $\mathrm{Na}_{2} \mathrm{WO}_{4}$, i.e, HWO in the reaction mixture.

As described in Section 3.5., the 135-min HT product was composed of predominantly noncrystalline (amorphous) nanoparticles, while the 140-min quenched sample contained $\mu \mathrm{m}$-sized FB BWO particles as well as nanoparticles but without unassembled BWO flakes (Fig. 7). If $\mu \mathrm{m}$-sized aggregates of nanoparticles containing bismuth and/or tungsten are formed at least just before the 140min HT reaction, there seems to be no reason for the aggregates to be decomposed back to nanoparticles when quenched. To interpret this sudden change between $135 \mathrm{~min}$ and $140 \mathrm{~min}$ of HT reaction, the above-mentioned BOx@HWO is presumed; $\mu \mathrm{m}$-sized BOx@HWO having fluidity (to be spherical and to be able to supply the bismuth component to the surface of BOx@HWO) is produced up to 135 min of HT reaction. The interaction between the BOx core and HWO shell might be a base-acid one, and quenching (or drying up) therefore decomposes the core-shell structure to give nanoparticles. It is hypothesized that at (or just before) the HT duration of $140 \mathrm{~min}$, i.e., when the temperature of the reaction mixture reaches a critical temperature, the second-step reaction of $\mathrm{BOx}$ and HWO starts to produce BWO flakes at the boundary of the BOx core and/or in HWO shell and is completed by ca. 180 min to leave ca. $80 \%$-crystalline BWO. During this second step, core size may be kept constant due to the solid BWO-flake shell except for the beginning of the BOx-HWO reaction. Lack of this size and morphology fixation by BWO flakes in the beginning leads to "starfruit-like" BOx particles as shown in Fig. 8; in the beginning, BOx particles with thin fragile BWO flakes are liberated but shrunk when recovered without interacting with other particles to liberate "starfruit-like" particles, the volume of which reflects the amount (concentration) of BOx in the core. With a longer HT duration, the thickness of the BWO-flake shell is increased not to be decomposed, i.e., a hard shell is produced. During the HT reaction between 135-180 min, the proportion of hard shell-covered particles is increased, resulting in a decrease in the proportion of nanoparticles (liberated from fragile shell-covered particles) in SEM images (Fig. 7). In the second step, following reaction releasing nitric acid may proceed, assuming $\mathrm{BO} 4$ is predominant in $\mathrm{BOx}$, to interpret observed decrease in $\mathrm{pH}$ qualitatively.

$$
\left[\mathrm{Bi}_{6} \mathrm{O}_{4}(\mathrm{OH})_{4}\right]\left(\mathrm{NO}_{3}\right)_{6}+3 \mathrm{H}_{2} \mathrm{WO}_{4} \rightarrow 3 \mathrm{Bi}_{2} \mathrm{WO}_{6}+6 \mathrm{HNO}_{3}+2 \mathrm{H}_{2} \mathrm{O}
$$

This is consistent qualitatively with the fact that $\mathrm{pH}$ was decreased in the period (Table 2).

As described in Section 3.1., the requisites for production of FB BWO particles with homogeneous shape and size are an acidic condition ( $\mathrm{pH}$ ca. 1) and $10 \mathrm{~mol} \%$-excess amount of tungsten source $\left(\mathrm{Na}_{2} \mathrm{WO}_{4}\right)$. It was reported in a previous paper [21] addition of sodium hydroxide to neutralize nitric acid released by hydrolysis of $\mathrm{Bi}\left(\mathrm{NO}_{3}\right)_{3}(\mathrm{pH}=\mathrm{ca} .4 .5)$ resulted in the formation of flake-stacked 
particles presumably without inner void spaces. Under such conditions, the bismuth component might be in the form of $\mathrm{Bi}(\mathrm{OH})_{3}$, not BOx, and thereby BOx@HWO could not be liberated in the HT reaction, leading to a simple reaction of $\mathrm{Bi}(\mathrm{OH})_{3}$ and $\mathrm{HWO}$ to form stacked flake-structured $\mathrm{BWO}$ particles. Wang et al. reported the influence of nitric acid concentration on the morphologies of BWO particles prepared by an $\mathrm{HT}$ reaction of $\mathrm{Bi}\left(\mathrm{NO}_{3}\right)_{3}$ and $\mathrm{Na}_{2} \mathrm{WO}_{4}$ in diluted nitric acid; the addition of nitric acid induced the production of particles in the form of stacked thick BWO platelets [40].

On the other hand, the addition of stoichiometric amount of $\mathrm{Na}_{2} \mathrm{WO}_{4}$ leads to the production of pseudo, i.e., partly broken, FB-structured BWO particles. As reported in a previous paper [25], the proportion of non-FB BWO particles was $73 \%$, while the proportion in the $0.55-\mathrm{W} / \mathrm{Bi}$ sample was only $28 \%$. Since initial $\mathrm{pH}$ value of the reaction mixtures before HT reaction with stoichiometric and $10 \mathrm{~mol} \%$-excess $\mathrm{Na}_{2} \mathrm{WO}_{4}$ were the same (1.1; entries 5 and 12 in Table 1), the difference in particle morphology might not be due to the difference in initial $\mathrm{pH}$. Thus, it is presumed that the BOx@HWO surface is not fully covered by HWO under the condition of stoichiometric- $\mathrm{Na}_{2} \mathrm{WO}_{4}$ feeding and thereby BWO flakes grow inhomogeneously. Actually, in the SEM images of 0.5-W/Bi FB BWO particles, there were interconnecting particles, which might share HWO layers with each other [21].

A paper from the authors' laboratory also showed change in morphology depending on the HT temperature in the range of $403-493 \mathrm{~K}(\mathrm{~W} / \mathrm{Bi}=0.55 ; 20 \mathrm{~h})$ [22]. It was suggested in the paper that crystalline-BWO content was increased with increase in HT temperature up to $433 \mathrm{~K}$ and was saturated at higher temperatures. At an HT temperature lower than $433 \mathrm{~K}$, reaction of BOx in the core and HWO in the shell might not be completed. On the other hand, crystalline-BWO content was still ca. 80\% (entry 6 in Table 1) even at a higher HT temperature, indicating that a part of BOx and a part of HWO remain unreacted in the core and shell, respectively, in an amorphous form. Another previous paper [22] showed change in the thickness of flakes with a slight increase in the diameter of FB particles; the average thickness of flakes was increased from ca. 25 to $50 \mathrm{~nm}$ linearly with increase in HT temperature. This could be interpreted by the slight increase in size of BOx@HWO. Generally, the higher the temperature is, the larger is the size of particles produced in the HT reaction. Assuming this trend is also applicable in the present case, increase in the diameter may induce a higher surface density of BWO if the thickness of the BWO-flake layer is constant regardless of temperature.

Zhang et al. reported preparation of FB and semi-FB BWO particles (called spherical and broken "flower-like" particles, respectively, in their paper) [26] and they proposed that spherical $\mu \mathrm{m}$ sized BWO-particle aggregates are produced by assembling of BWO nanoparticles of a layered crystal structure and then all of the incorporated nanoparticles undergo Ostwald ripening to form plate-like crystallites ("nanoplates") that are larger than the original nanoparticles but smaller than the resultant FB BWO particles; production of FB and/or semi-FB structures was attributed to "self-organization of the in situ-formed nanoplates". However, the reason why BWO nanoparticles are aggregated into $\mu \mathrm{m}$-sized BWO particles in the first step without undergoing Ostwald ripening to produce larger particles and the reason why "self-organization of nanoplates" produces FB particles of higher surface 
energy were not clarified. It is not known whether the authors recognized the hollow structure of their FB particles since no such description was found (a scheme in the paper might suggest). Considering their experimental conditions that were similar to those of the present study, their FB BWO particles might have inner void spaces, but their proposed mechanism does not seem to sufficiently explain the construction of a hollow FB structure thoroughly.

\subsection{Photocatalytic activity of FB BWO particles}

Table 2 shows photocatalytic activity of samples for carbon-dioxide liberation from an aerobic aqueous solution of acetic acid. First, contrary to the authors' expectation, the sample recovered from the reaction mixture without HT reaction (entry 1 in Table 2) showed the highest photocatalytic activity, which was comparable to or slightly lower than that of commercial titania samples (Showa Denko Ceramics FP-6: $77 \mu \mathrm{mol} \mathrm{h}^{-1}$ and Evonik P25: $77 \mu \mathrm{mol} \mathrm{h}^{-1}$ ), among the samples shown in Table 2. Since the XRD pattern of this sample gave little information on the contents; $88 \%$ content was non-crystalline phase, which may contain amorphous solids and water, but the composition of this amorphous solid could not be clarified. In other words, the samples prepared without HT reaction or with short HT reaction $(<145 \mathrm{~min}$ ) cannot be assigned to BWO, bismuth tungstate $\left(\mathrm{Bi}_{2} \mathrm{WO}_{6}\right)$, and they might be "non-BWO" samples. The reason for this higher activity is unclear, and studies on the characteristics and photocatalytic activity of this sample are now under way. Activities of samples prepared by HT reaction of duration in the range of 145-300 min were lower than those of samples with a longer HT duration (ca. $20 \mu \mathrm{mol} \mathrm{h}^{-1}$, corresponding to ca. $25 \%$ of activities of commercial titania samples). The increase in activity with increase in HT duration is possibly related to the BWO-crystalline (or non-crystalline) content, suggesting that only crystalline BWO is active for the photocatalytic reaction of acetic-acid decomposition. Detailed analysis of photocatalytic activities with other physical properties, e.g., specific surface area [22], and investigation of the effect of particle morphology on photocatalytic activity as well as the effect of loaded co-catalysts such as graphene [41] on photocatalytic activity is now under way and the results will be reported in the near future.

\section{Conclusions}

The hollow structure of FB BWO particles prepared by HT reaction of an aqueous suspension containing $\mathrm{Bi}\left(\mathrm{NO}_{3}\right)_{3}$ and $10 \mathrm{~mol} \%$ stoichiometric excess of $\mathrm{Na}_{2} \mathrm{WO}_{4}$ was supported by FE-SEM observation of sonicated samples, FE-SEM observation of FIB-milled samples and UHV-TEM analysis. A model of the formation mechanism, in which once-formed BOx@HWO core-shell droplets (or fluidic particles) are converted into hollow FB BWO particles by the reaction of BOx and HWO migrating from the inside and outside, respectively, was proposed for the first time as one of the most plausible interpretations being consistent with almost all of the results of the present study and previous studies on FB BWO preparations. Ma and coworkers reported the preparation of solid (with no suggestion of its hollow structure) FB-structured bismuth oxyiodide (BiOI) particles and their topochemical conversion into hollow FB BWO particles [42]. Considering the proposed mechanism, 
it seems probable that (i) the BiOI particles having inner void spaces were produced by a mechanism similar to that proposed here and converted into FB BWO without morphological change (topochemical) or (ii) the BiOI particles have a solid structure and were converted to FB BWO particles by a mechanism similar to that proposed here.

In general, it is believed that HT reactions proceed through growth of particles via the Ostwald ripening mechanism, i.e., dissolution of smaller particles to be deposited on larger particles. In this case, particle size is regulated by particle solubility depending on the size of particles, and the product particles may have the lowest surface energy, e.g., spherical particles. A hidden (not explicit) condition for the Ostwald-ripening mechanism is that small particles with the same composition as that of the final product are produced at the beginning of the reaction. In the present study, however, it is not reasonable to assume that small BWO particles (or flakes) are produced by the reaction of bismuth and tungsten components and that these small particles then grow via the Ostwald-ripening mechanism since such processes cannot explain (1) why the hollow structure and FB BWO particles do not seem to have the lowest surface energy and (2) why FB BWO-particles suddenly appear during the HT process. A mechanism including fluidic BOx@HWO as a key intermediate could be an alternative HT-reaction mechanism, especially for the reaction of acid and base components to liberate practically insoluble products. In the proposed mechanism, HT conditions would provide the reaction components with higher solubility, but the Ostwald-ripening mechanism might not be included. To the best of the authors' knowledge, such a non-conventional HT-reaction mechanism including intermediacy of core shell-structured liquid droplets has not been reported so far.

The information presented here for the characteristic shaped-particle fabrication may be useful for the development of shape-controlled functional powder materials. Further studies on the detailed mechanism, especially the initial BOx@HWO formation and how the size of BOx@HWO is regulated, as well as on the correlation of photocatalytic activities with particle morphology are now in progress.

\section{Acknowledgements}

The authors are grateful to Professor Masato Hashimoto (Wakayama University, Japan) for kind guidance and suggestions regarding the structure and reaction of tungstic acid/tungstate in acidic solutions. A part of the analyses was carried out with JEOL JSM-7400F and JSM-6360LA electron microscopes in the OPEN FACILITY, Hokkaido University Sousei Hall.

\section{References}

[1] A. Fujishima, X. Zhang, D. A. Tryk, Int. J. Hydrogen Energy 32 (2007) 2664- 2672.

[2] A. Fujishima, X. T. Zhang, D. A. Tryk, Surf. Sci. Rep. 63 (2008) 515-582.

[3] B. Ohtani, J. Photochem. Photobiol., C: Photochem. Rev. 11, 157-178 (2010).

[4] B. Ohtani, Electrochemistry 82 (2014) 414-425.

[5] D. E. Scaife, Sol. Energy 25 (1980) 41-54. 
[6] K. Nishijima, B. Ohtani, X. Yan, T.-a. Kamai, T. Chiyoya, T. Tsubota, N. Murakami and T. Ohno, Chem. Phys. 339 (2007) 64-72.

[7] M.V. Dozzi and E. Selli, J. Photochem. Photobiol. C: Photochem. Rev. 14 (2013) 13-28.

[8] R. Abe, J. Photochem. Photobiol., C: Photochem. Rev. 11 (2010) 179-209.

[9] K. Maeda, J. Photochem. Photobiol., C: Photochem. Rev. 12 (2011) 237-268.

[10] K. Domen, A. Kudo, M. Shibata, A. Tanaka, K.-I. Maruya and T. Onishi, J. Chem. Soc., Chem. Commun. (1986) 1706-1707.

[11] B. Ohtani, F. Amano, T. Yasumoto, O.-O. Prieto-Mahaney, S. Uchida, T. Shibayama and Y. Terada, Top. Catal. 53 (2010) 455-461.

[12] E. Grabowska, M. Diak, M. Marchelek and A. Zaleska, Appl. Catal. B: Environ. 156-157 (2014) 213-235.

[13] Z. Wei, E. Kowalska, J. Verrett, C. Colbeau-Justin, H. Remita and B. Ohtani, Nanoscale 7 (2015) 12392-12404.

[14] M. Maisano, M. V. Dozzi and E. Selli, J. Photochem. Photobiol. C: Photochem. Rev. 28 (2016) 29-43.

[15] T. Liu, X. Zhou, M. Dupuisc and C. Li, Phys. Chem. Chem. Phys., 17 (2015) 2350323510.

[16] R. Li, F. Zhang, D. Wang, J. Yang, M. Li, J. Zhu, X. Zhou, H. Han and C. Li, Nature Commun. 4 (2013) 1432 (1-7).

[17] B. Ohtani, S. Ikeda, H. Nakayama and S.-i. Nishimoto, Phys. Chem. Chem. Phys. 2 (2000) 5308-5313.

[18] F. Amano, T. Yasumoto, T. Shibayama, S. Uchida and B. Ohtani, Appl. Catal. B: Environ. 89 (2009) 583-589.

[19] K. Domen, Y. Ebina, S. Ikeda, A. Tanaka, J. N. Kondo and K. Maruya, Catal. Today 28 (1996) 167-174.

[20] F. Amano, K. Nogami, R. Abe and B. Ohtani, Chem. Lett. 36 (2007) 1314-1315.

[21] F. Amano, K. Nogami, R. Abe and B. Ohtani, J. Phys. Chem. C 112 (2008) 9320-9326.

[22] F. Amano, K. Nogami and B. Ohtani, Langmuir 26 (2010) 7174-7180.

[23] F. Amano, K. Nogami and B. Ohtani, J. Phys. Chem. C 113 (2009) 1536-1542.

[24] K. S. Knight, Mineral. Magazine 56 (1992) 399-409.

[25] H. Hori, M. Takase, F. Amano and B. Ohtani, Chem. Lett. 44 (2015) 1723-1725.

[26] L. H. Zhang, W. Z. Wang, Z. G. Chen, L. Zhou, H. I. Xu and W. Zhu, J. Mater. Chem. 17 (2007) 2526-2532.

[27] L. S. Zhang, W. Z. Wang, L. Zhou and H. L. Xu, Small 3 (2007) 1618-1625.

[28] F. Izumi and K. Momma, Solid State Phenom., 130 (2007) 15-20.

[29] H. Kominami, Y. Ishii, M. Kohno, S. Konish, Y. Kera and B. Ohtani, Catal. Lett. 91 (2003) 41-47.

[30] H. Kominami, J.-i. Kato, M. Kohno, Y. Kera and B. Ohtani, Chem. Lett. 25 (1996) 1051- 
1052.

[31] B. Ohtani, O.-O. Prieto-Mahaney, F. Amano, N. Murakami and R. Abe, J. Adv. Oxidat. Tech. 13 (2010) 247-261.

[32] S.-i. Nishimoto, B. Ohtani and T. Kagiya, J. Chem. Soc., Faraday Trans. 181 (1985) 24672474.

[33] B. Ohtani, M. Kakimoto, S.-i. Nishimoto and T. Kagiya, J. Photochem. Photobiol. A: Chem. 70 (1993) 265-272.

[34] H. Hori, M. Takashima, M. Takase and B. Ohtani, to be submitted.

[35] F. Lazarini, Thermochim. Acta .46 (1981) 53-55

[36] Y. He, Y. Zhang, H. Huang, N. Tian, and Y. Luo, Inorg. Chem. Commun. 40 (2014) 55-58

[37] J. J. Hastings and O. W. Howarth, J. Chem. Soc., Dalton Trans. (1992) 209-214.

[38] H. Huang, Y. He, X. Li, M. Li, C. Zeng, F. Dong, X. Du, T. Zhang and Y. Zhang, J. Mater. Chem. A 3 (2015) 24547-24556.

[39] M. Gotić, S. Popović and S. Musić, Mater. Lett. 61 (2007) 709-714.

[40] W. Wang, M. Guo, D. Lu, W. Wang and Z. Fu, Crystals 6 (2016) 75 (1-11).

[41] S. Gupta and V. Subramanian, ACS Appl. Mater. Interfaces 6 (2014) 18597-18608.

[42] D. K. Ma, S.M. Zhou, X. Hu, Q. R. Jiang and S. M. Huang, Mater. Chem. Phys. 140 (2013) $11-15$. 
Table 1

Volume, $\mathrm{pH}$ and molar amount of protons in the solutions (suspensions) for FB-BWO preparation/control experiments and crystalline/noncrystalline (nc) composition of resulting recovered/dried-up solid products.

\begin{tabular}{|c|c|c|c|c|c|c|c|}
\hline $\begin{array}{l}\text { en- } \\
\text { try }\end{array}$ & $\begin{array}{c}\text { HT } \\
\text { dura- } \\
\text { tion }{ }^{\mathrm{a}} / \mathrm{h}\end{array}$ & component (/mmol) & $\begin{array}{l}\text { volume } \\
\text { /mL }\end{array}$ & $\mathrm{pH}$ & $\begin{array}{c}\mathrm{H}^{+} \\
/ \mathrm{mmol}\end{array}$ & $\begin{array}{c}\text { crystalline } \\
(\%)\end{array}$ & $\begin{array}{l}\mathrm{nc} \\
(\%)\end{array}$ \\
\hline 1 & 0 & $\mathrm{Bi}\left(\mathrm{NO}_{3}\right)_{3}(5.0)$ & 10 & $0.27-0.28$ & $5.3-5.2$ & $n d^{b}$ & $\mathrm{nd}^{\mathrm{b}}$ \\
\hline 3 & 0 & $\mathrm{Na}_{2} \mathrm{WO}_{4}(2.75)$ & 40 & 7.8-7.9 & $<0.1$ & $n d^{b}$ & $\mathrm{nd}^{\mathrm{b}}$ \\
\hline 4 & 0 & $\mathrm{Na}_{2} \mathrm{WO}_{4}(2.75)+\mathrm{HNO}_{3}(15.0)+\mathrm{NaOH}^{\mathrm{d}}$ & 70 & 1.1 & 5.6 & $(\text { yellow solid })^{\mathrm{b}}$ & $\mathrm{nd}^{\mathrm{b}}$ \\
\hline 7 & 0 & $\mathrm{Bi}\left(\mathrm{NO}_{3}\right)_{3}(5.0)+\mathrm{NaOH}(5)$ & 70 & 0.82 & 10.6 & $\mathrm{nd}^{\mathrm{b}}$ & $\mathrm{nd}^{\mathrm{b}}$ \\
\hline 8 & 0 & $\mathrm{Bi}\left(\mathrm{NO}_{3}\right)_{3}(5.0)+\mathrm{NaOH}(5.5)$ & 70 & 0.83 & 10.4 & $\mathrm{nd}^{\mathrm{b}}$ & $\mathrm{nd}^{\mathrm{b}}$ \\
\hline 9 & 20 & $\mathrm{Bi}\left(\mathrm{NO}_{3}\right)_{3}(5.0)+\mathrm{NaOH}(5.5)$ & 70 & 1.1 & 5.6 & $\left(\mathrm{BO}^{\mathrm{h}}\right)^{\mathrm{i}}$ & $\mathrm{nd}^{\mathrm{b}}$ \\
\hline 10 & 0 & $\mathrm{HNO}_{3}(15.0)+\mathrm{NaOH}(5.5)$ & 70 & 0.87 & 9.4 & $-^{\mathrm{c}}$ & — $^{\mathrm{c}}$ \\
\hline
\end{tabular}

a Period of hydrothermal treatment at $433 \mathrm{~K}{ }^{\mathrm{b}} \mathrm{Not}$ recovered and/or not determined. $\quad{ }^{\mathrm{c}}$ No solid products. ${ }^{\mathrm{d}}$ Added to adjust $\mathrm{pH}$ to be 1.1 . ${ }^{e}\left[\mathrm{Bi}_{6} \mathrm{O}_{5}(\mathrm{OH})_{3}\right]\left(\mathrm{NO}_{3}\right)_{5} \cdot 3 \mathrm{H}_{2} \mathrm{O} . \quad{ }^{\mathrm{f}}\left[\mathrm{Bi}_{6} \mathrm{O}_{4}(\mathrm{OH})_{4}\right]\left(\mathrm{NO}_{3}\right)_{6} \cdot \mathrm{H}_{2} \mathrm{O}$. $\quad$ gNon crystalline-phase content was not determined and crystalline content is shown

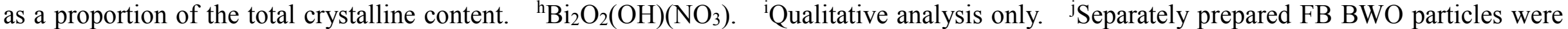
added. ${ }^{\mathrm{k}}$ Content of water was ca. $1 \%$ based on the results of thermogravimetry. 


\section{Table 2}

Crystalline composition of HT products recovered by quenching and final $\mathrm{pH}$ of the reaction mixture.

\begin{tabular}{|c|c|c|c|c|c|c|c|}
\hline \multirow{2}{*}{$\begin{array}{l}\text { HT dura- } \\
\text { tion } / \mathrm{min}\end{array}$} & \multicolumn{5}{|c|}{ composition (\%) } & \multirow{2}{*}{$\mathrm{pH}^{\mathrm{g}}$} & \multirow{2}{*}{$\begin{array}{l}\text { activity }^{\mathrm{h}} \\
/ \mu \mathrm{mol} \mathrm{h}{ }^{-}\end{array}$} \\
\hline & $\mathrm{BWO}^{\mathrm{b}}$ & $\mathrm{BO}^{\mathrm{c}}$ & $\mathrm{BO} 4^{\mathrm{d}}$ & $\mathrm{HWO}^{\mathrm{e}}$ & $n c^{f}$ & & \\
\hline $0^{\mathrm{i}}$ & $<0.5$ & $<0.5$ & 11 & 1 & 88 & 1.11 & 55 \\
\hline 135 & 1 & 1 & 3 & 1 & 94 & 0.99 & 10 \\
\hline 140 & 32 & 4 & 0 & 0 & 64 & 1.01 & 4 \\
\hline 145 & 69 & 6 & 0 & 2 & 23 & 1.00 & 4 \\
\hline 150 & 65 & 9 & 0 & 1 & 25 & 1.01 & 11 \\
\hline 165 & 76 & 1 & 1 & 1 & 21 & 0.98 & 10 \\
\hline 180 & 78 & 1 & 3 & 0 & 18 & 0.97 & 11 \\
\hline 300 & 87 & 1 & 3 & 0 & 9 & 0.96 & 10 \\
\hline 600 & 88 & 2 & 0 & 0 & 10 & 0.95 & 20 \\
\hline 900 & 76 & 0 & 0 & 0 & 24 & 0.95 & 16 \\
\hline $1200^{j}$ & 80 & 1 & 3 & 0 & 16 & 0.96 & 18 \\
\hline
\end{tabular}

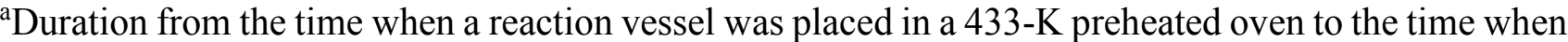
the vessel was taken out and quenched in ice-cold water. ${ }^{\mathrm{b}} \mathrm{Bi}_{2} \mathrm{WO}_{6} . \quad{ }^{c}\left[\mathrm{Bi}_{6} \mathrm{O}_{5}(\mathrm{OH})_{3}\right]\left(\mathrm{NO}_{3}\right)_{5} \cdot 3 \mathrm{H}_{2} \mathrm{O}$. ${ }^{\mathrm{d}}\left[\mathrm{Bi}_{6} \mathrm{O}_{4}(\mathrm{OH})_{4}\right]\left(\mathrm{NO}_{3}\right)_{6} \cdot \mathrm{H}_{2} \mathrm{O}$. ${ }^{\mathrm{e}} \mathrm{HWO}$. ${ }^{\mathrm{f}}$ Non-crystalline component. ${ }^{\mathrm{g}}$ Final $\mathrm{pH}$ of the reaction mixture. ${ }^{\text {h}}$ Rate of carbon-dioxide liberation in photocatalytic oxidative decomposition of acetic acid in an aerobic aqueous suspension. ${ }^{\mathrm{i}}$ No HT treatment. ${ }^{\mathrm{j}}$ No quenching. 


\section{Figure captions}

Fig. 1. FE-SEM images of FB BWO particles $(\mathrm{W} / \mathrm{Bi}=0.55)$ of different magnifications.

Fig. 2. FE-SEM images of FB BWO particles $(\mathrm{W} / \mathrm{Bi}=0.50)(\mathrm{a}-\mathrm{c})$ before sonication and after 90min sonication under (d) acidic, (e) neutral and (f) basic conditions.

Fig. 3. FE-SEM images of $(a, b) F B$ BWO particles $(\mathrm{W} / \mathrm{Bi}=0.55)$ after treatment with aqueous sodium hydroxide and $(\mathrm{c}, \mathrm{d}) \mathrm{FB}$ BWO particles $(\mathrm{W} / \mathrm{Bi}=0.50)$ without treatment at different magnifications.

Fig. 4. Cross-sectional FE-SEM images of FB BWO particles $(\mathrm{W} / \mathrm{Bi}=0.55)$ after FIB milling.

Fig. 5. A TEM image and observed/simulated cross-sectional thickness profiles. Thin solid lines are simulated thickness profiles of spherical hollow particles with shells of homogeneous density with different thicknesses $(55 \%, 50 \%, 45 \%$ and $35 \%$ of the outer radius from top to bottom).

Fig. 6 TEM images of solid samples $(\mathrm{W} / \mathrm{Bi}=0.55)$ recovered by quenching after HT reaction for (a) $140 \mathrm{~min}$, (b) $300 \mathrm{~min}$, (c) $600 \mathrm{~min}$ and (d) $900 \mathrm{~min}$. Particles smaller than $2 \mu \mathrm{m}$ were chosen. A hollow structure was suggested for the particles shown by arrows.

Fig. 7. Solid samples $(\mathrm{W} / \mathrm{Bi}=0.55)$ recovered by quenching after HT reactions for (a) $135 \mathrm{~min}$, (b) $140 \mathrm{~min}$, (c) $145 \mathrm{~min}$ and (d) $150 \mathrm{~min}$. Specimens were prepared via back-side sampling. White bars correspond to $10 \mu \mathrm{m}$.

Fig. 8. SEM (left) and EDS elemental mapping (right) images of (a-e) solid samples containing starfruit-like particles (shown as green particles in elemental mapping images) $(\mathrm{W} / \mathrm{Bi}=0.55)$ recovered by quenching after $\mathrm{HT}$ reaction for $135 \mathrm{~min}$ and (f) $\mathrm{FB} \mathrm{BWO}(\mathrm{W} / \mathrm{Bi}=0.55$ ). White bars correspond to $3 \mu \mathrm{m}$. In elemental mapping, green, red and blue correspond to bismuth, tungsten and carbon, respectively. Tungsten/bismuth atomic ratios in the images were (a) 3.1, (b) 3.1, (c) 2.7, (d) 2.5, (e) 2.4 and (f) 1.9. Specimens of quenched samples were prepared via back-side sampling $(\mathrm{a}-\mathrm{b})$ and front-side sampling $(\mathrm{c}-\mathrm{e})$.

Fig. 9. FE-SEM images of solid products obtained by $(\mathrm{a}, \mathrm{b})$ drying of a suspension $(\mathrm{W} / \mathrm{Bi}=0.55)$ for HT reaction (entry 5 in Table 1) and (c,d) HT reaction of a suspension containing bismuth nitrate and sodium hydroxide (entry 9 in Table 1).

Fig. 10. Schematic representation of the proposed mechanism of FB BWO-particle production. 


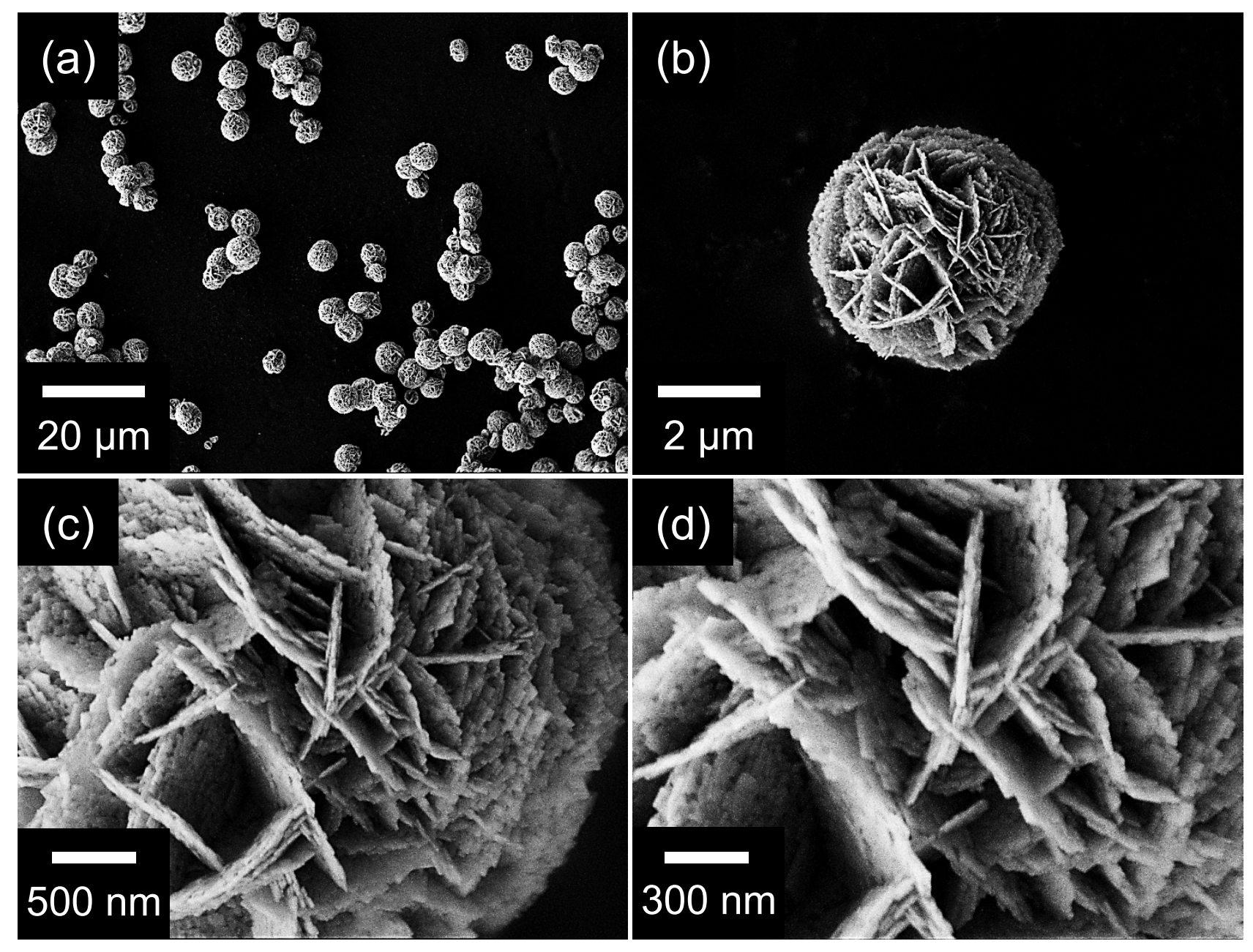

Fig. 1 


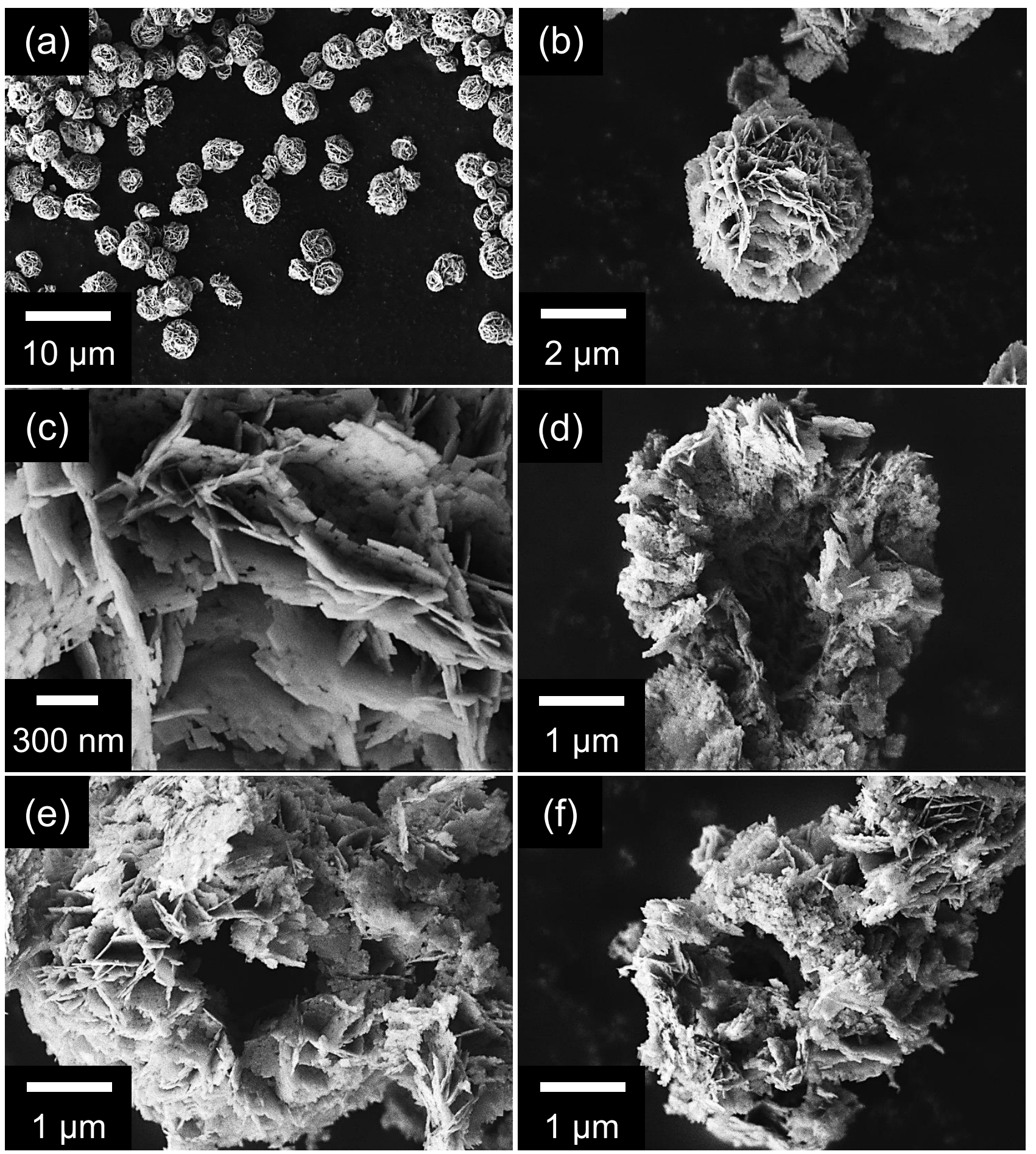

Fig. 2 


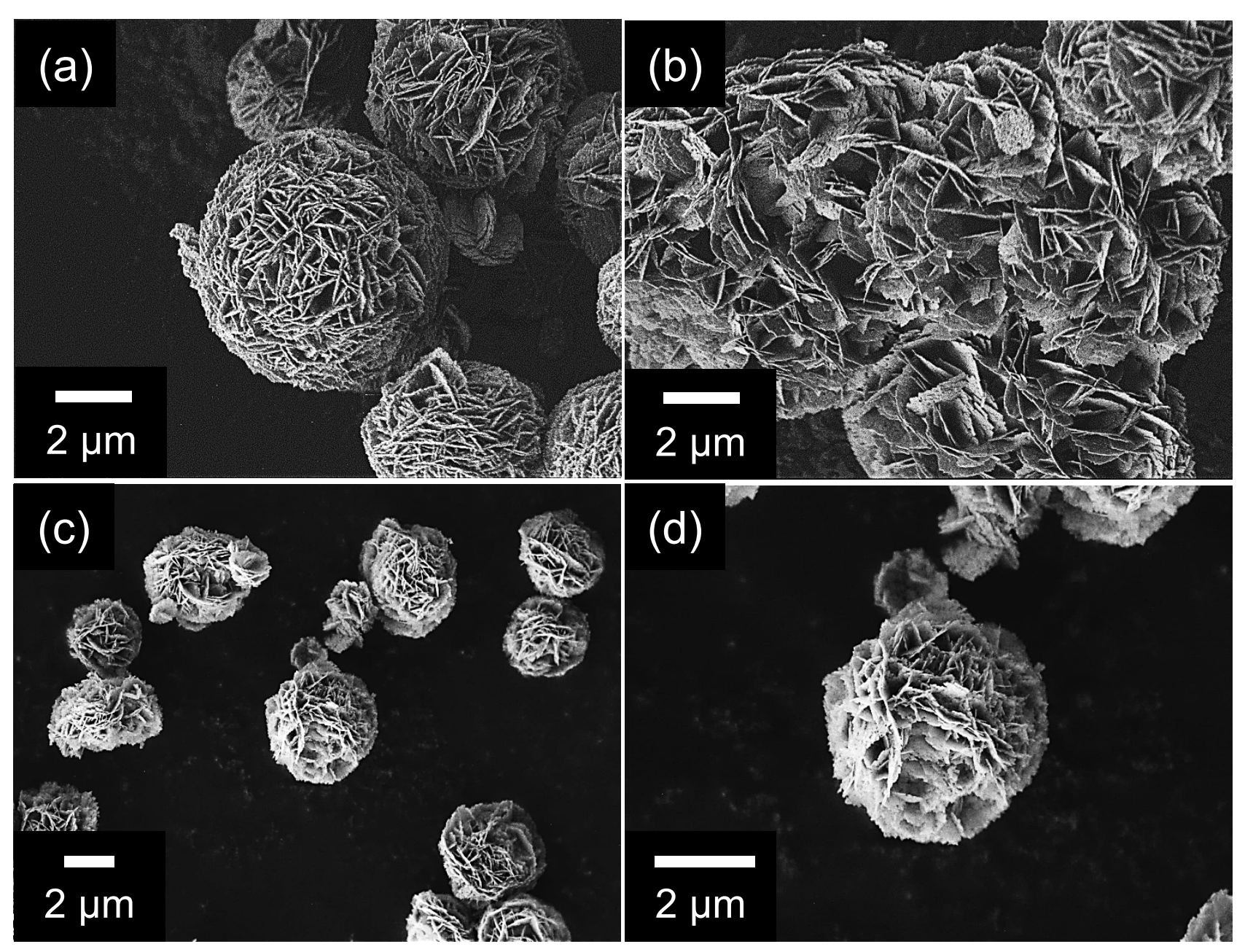

Fig. 3 


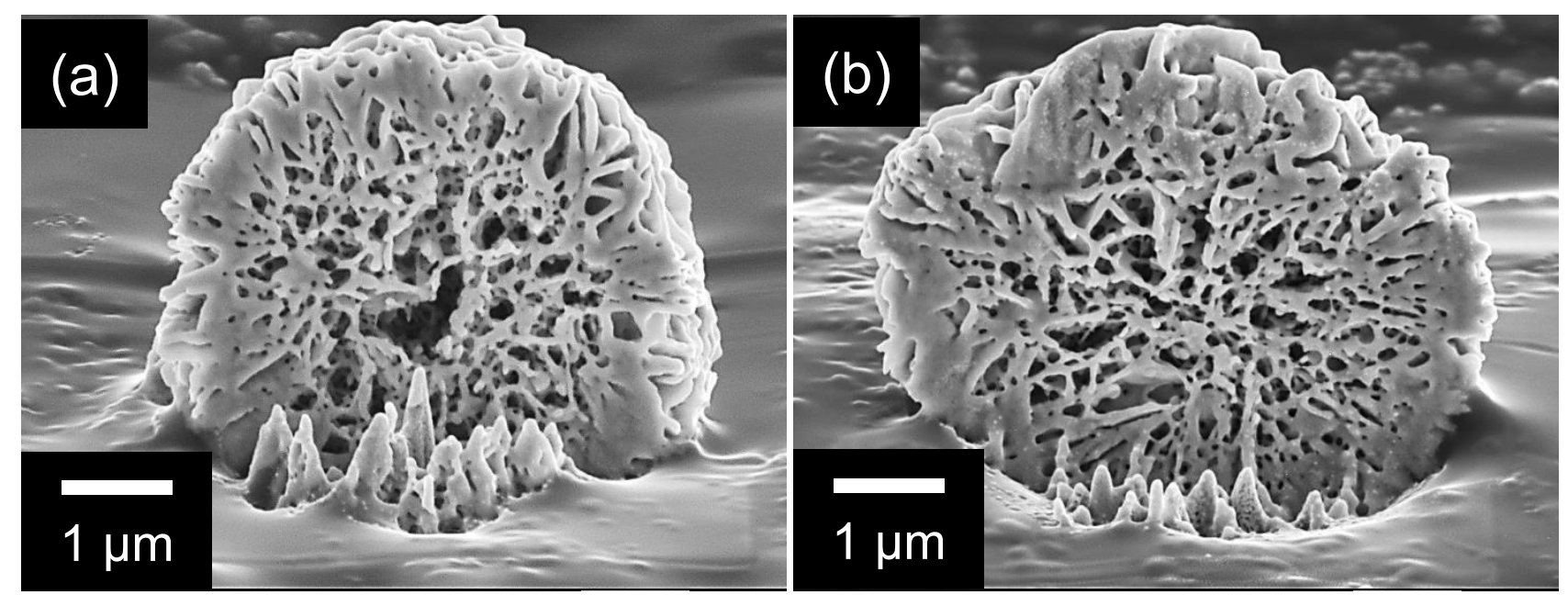

Fig. 4 

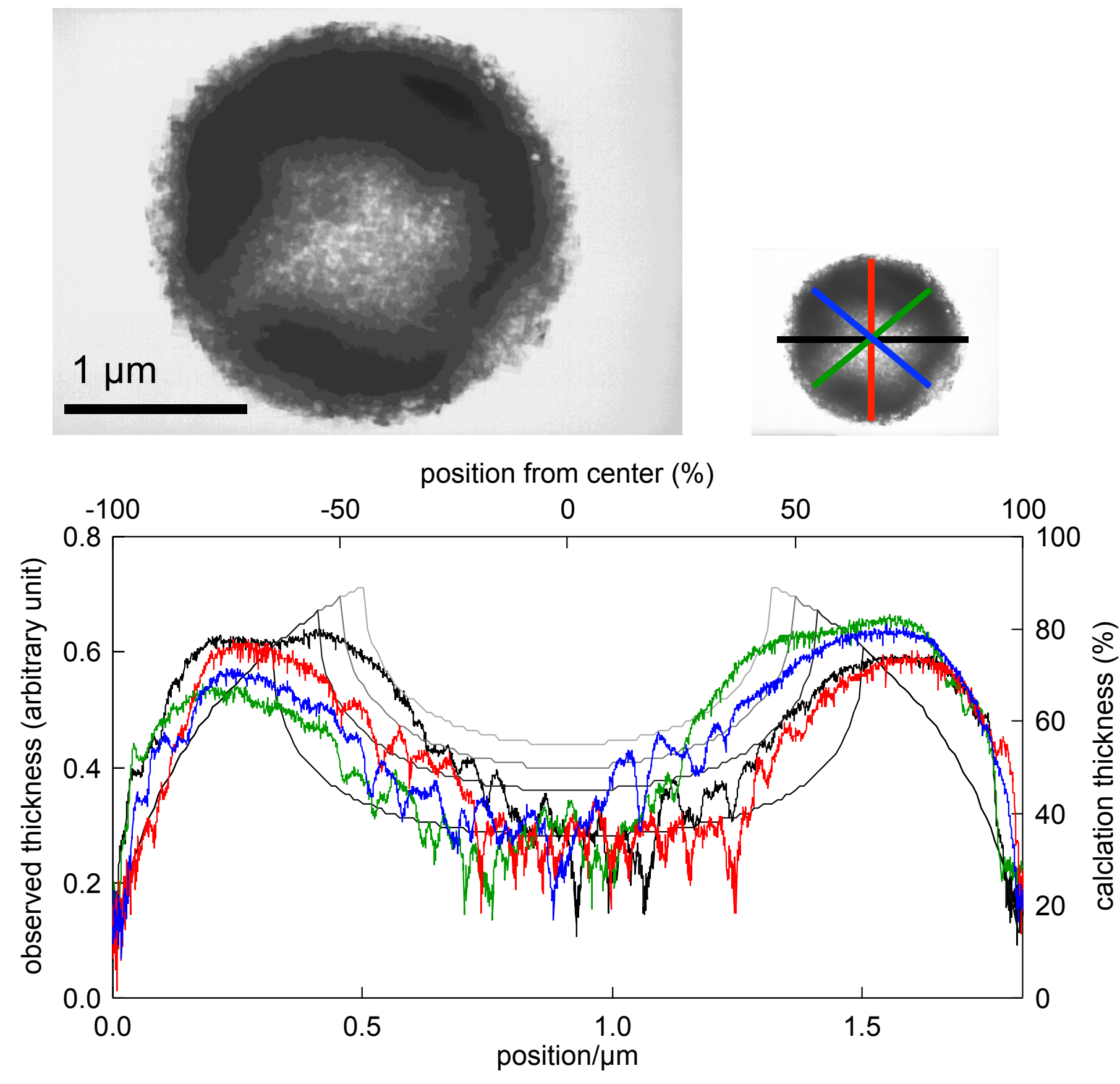

Fig. 5 
(a)

- valum

(b)
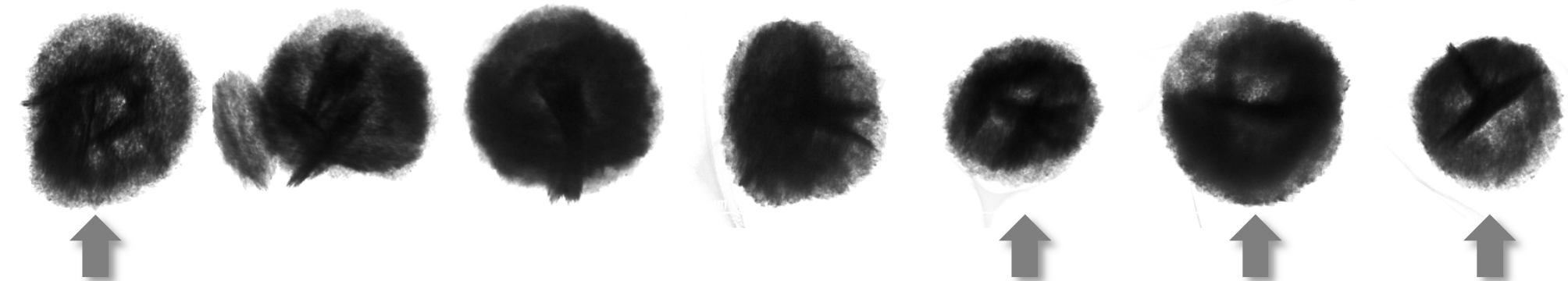

(c)
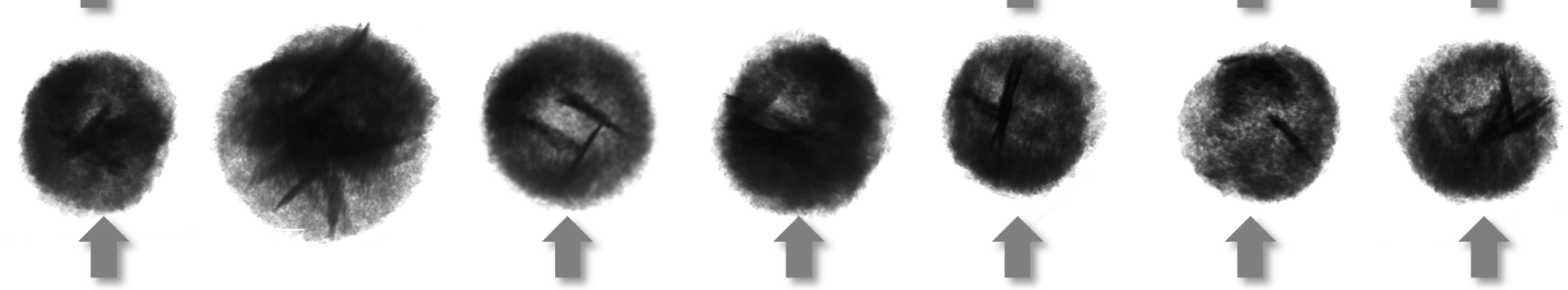

(d)
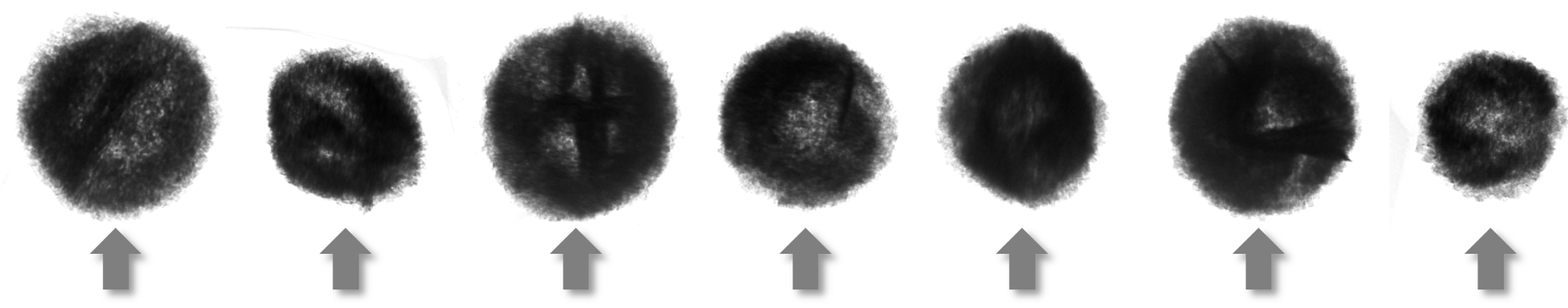

Fig. 6 


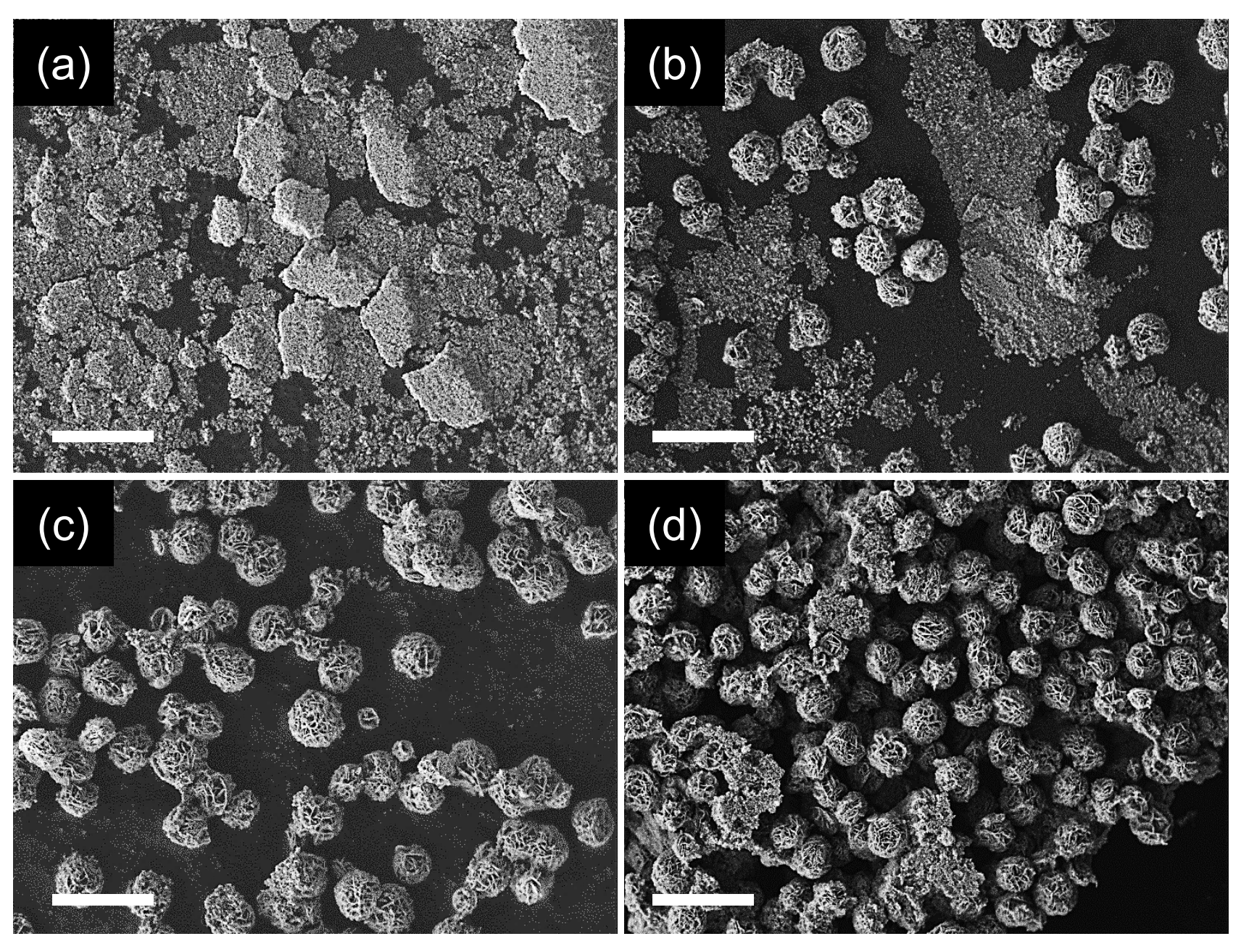

Fig. 7 


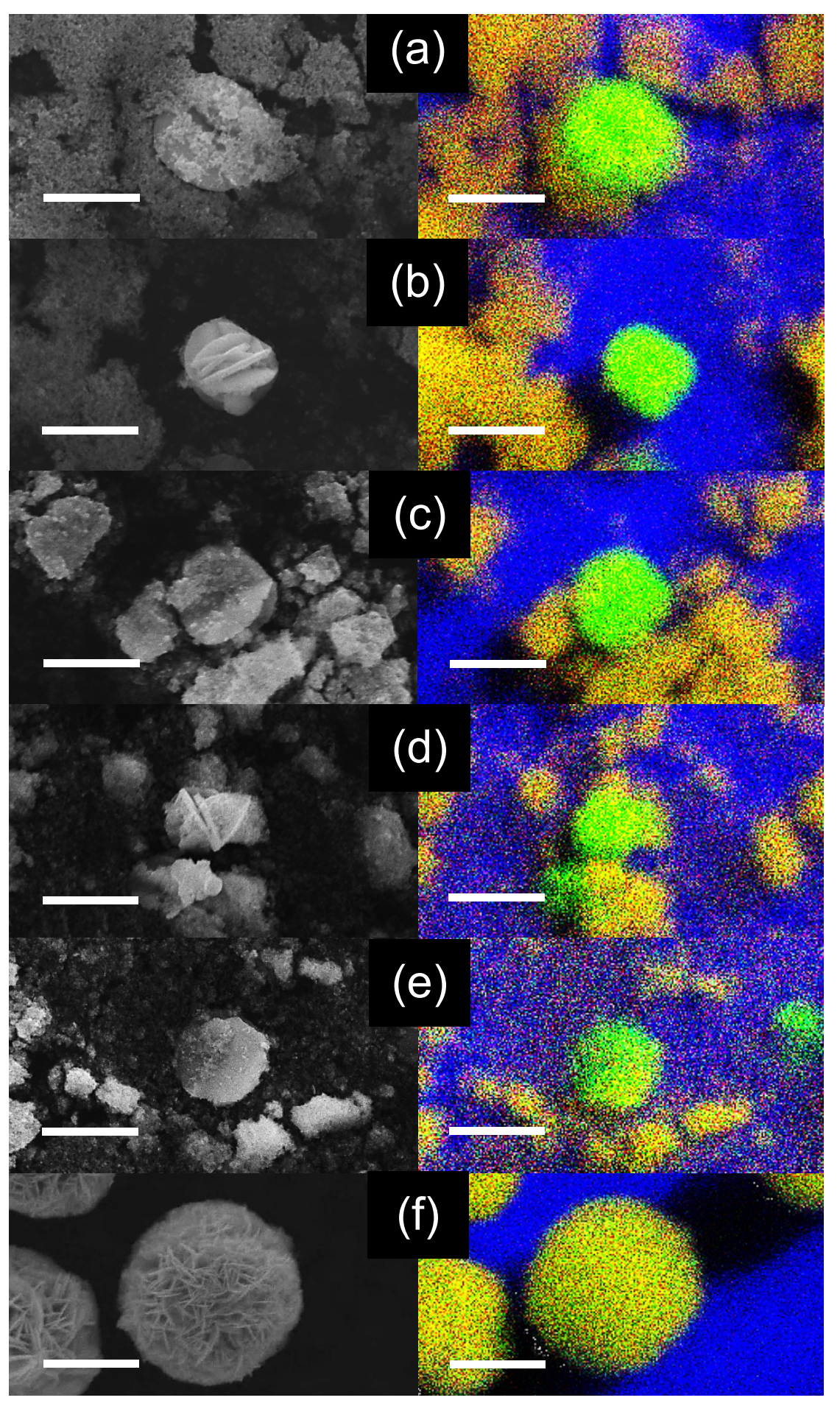

Fig. 8 


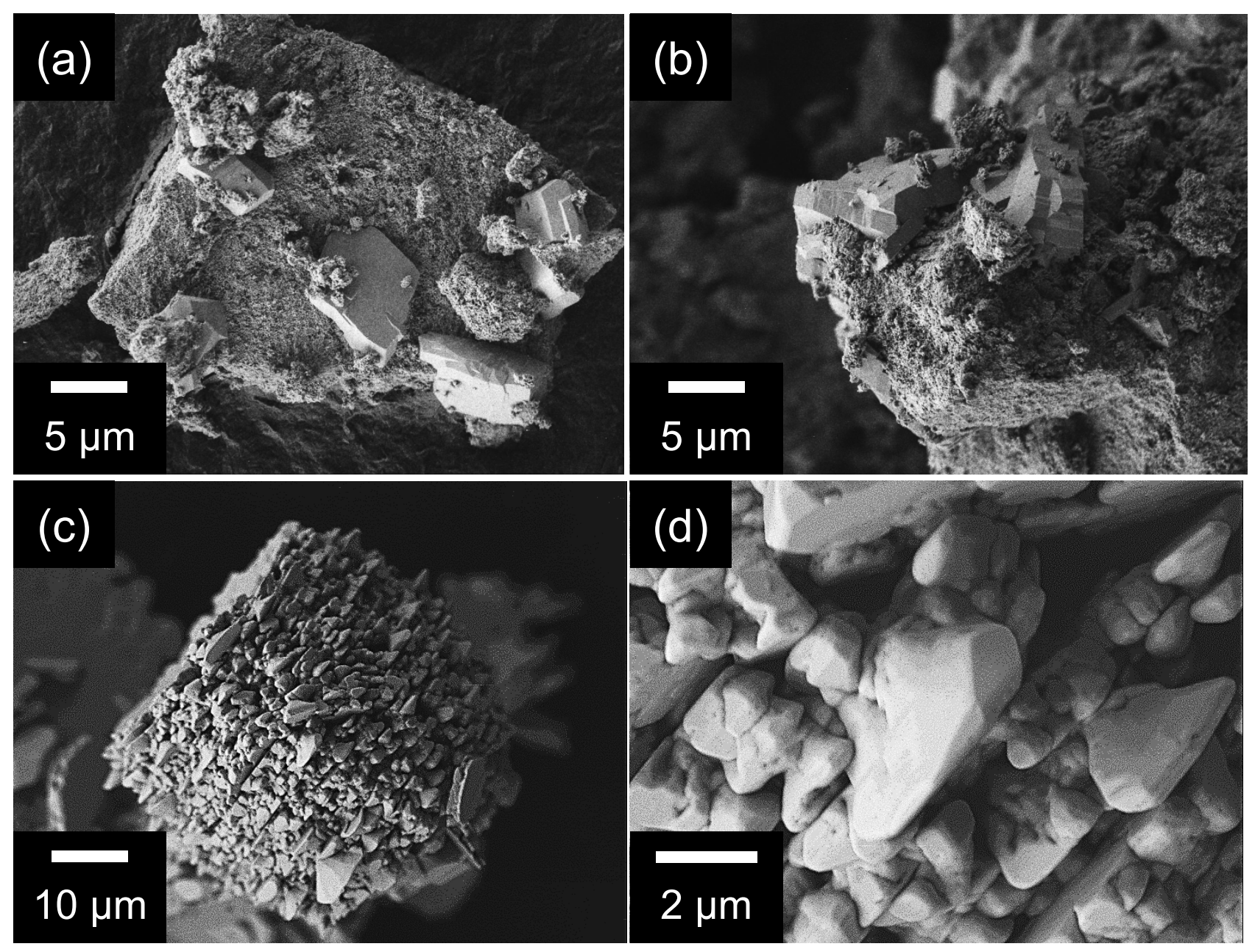

Fig. 9 

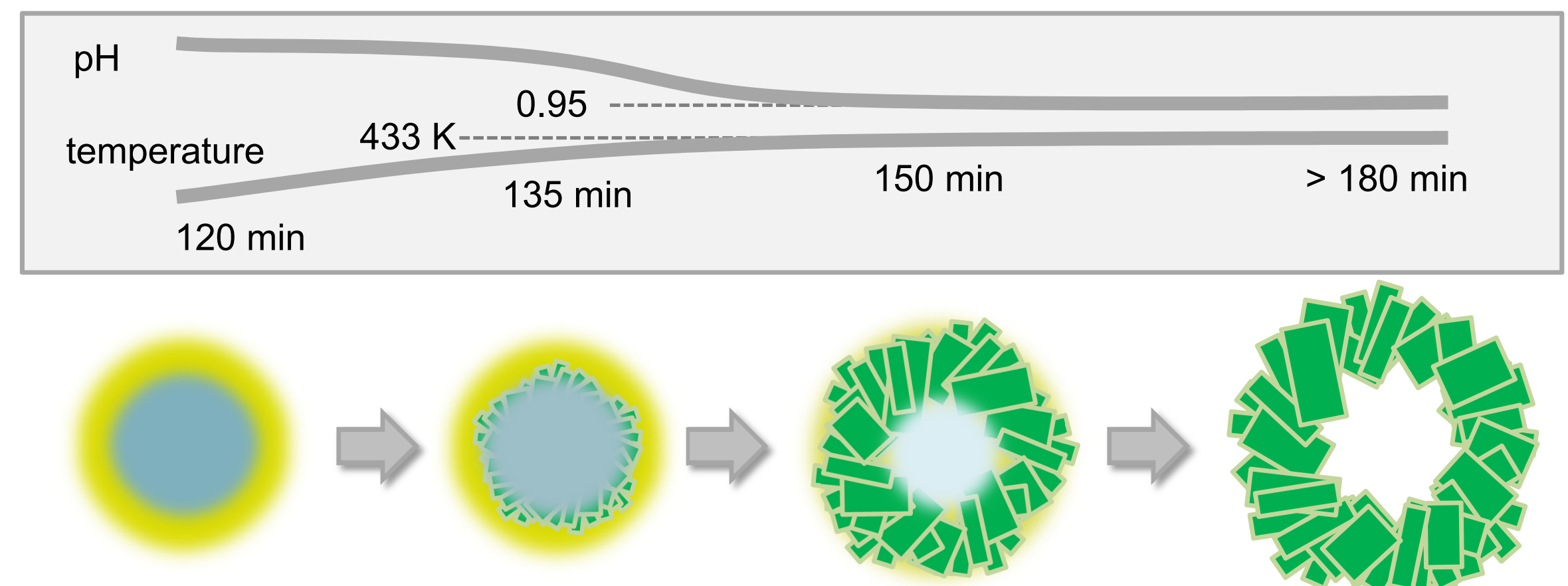

conc. BOx aq. core HWO shell=BOx@HWO

conc, BOx aq. core BWO flakes/HWO shell dil. BOx aq. core BWO flakes/HWO shell

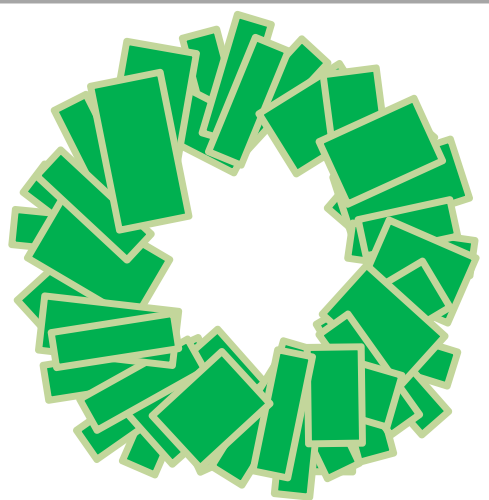

BWO-flake shell $=$ (hollow) FB BWO

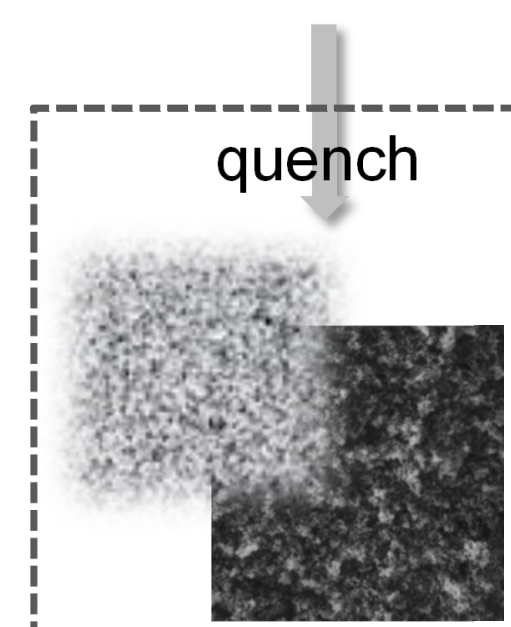

nanoparticles

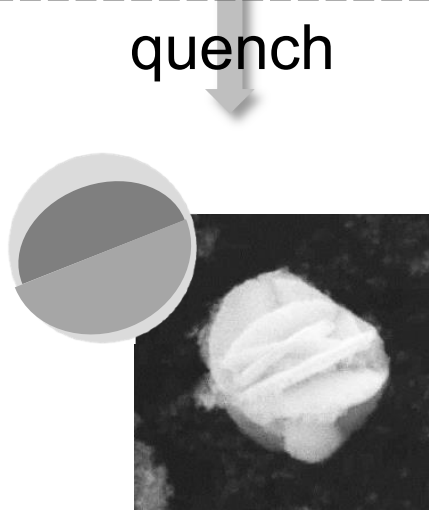

starfruit BOx particle

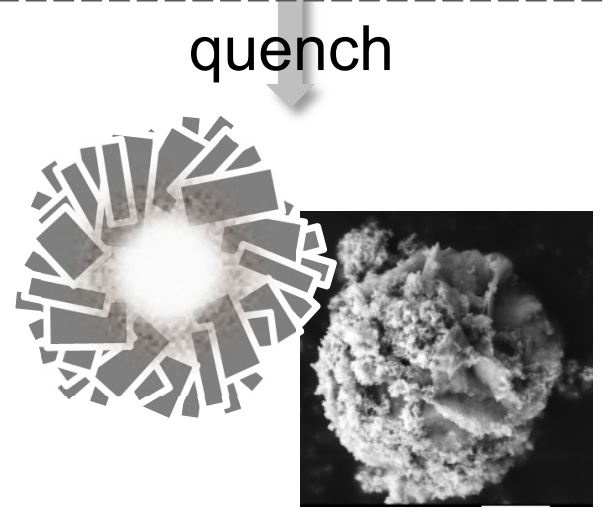

FB BWO/BOx + HWO

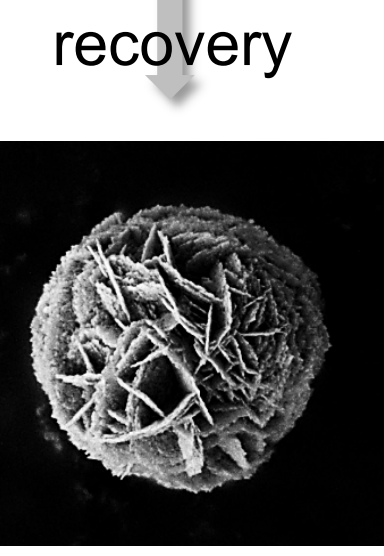

